\title{
Dynamics of a lattice gas system of three species
}

\author{
Yuanshi Wang, Hong $\mathrm{Wu}^{*}$ and Junhao Liang \\ School of Mathematics and Computational Science \\ Sun Yat-sen University, Guangzhou 510275, P.R.China
}

\begin{abstract}
This paper considers a mutualism system of three species in which each species provides resource for the next one in a one-directional loop, while there exists spatial competition among them. The system is characterized by a lattice gas model and the cases of obligate mutualisms, obligate-facultative mutualisms and facultative mutualisms are considered. Using dynamical systems theory, it is shown that (i) the mutualisms can lead to coexistence of species; (ii) A weak mutualism or an extremely strong mutualism will result in extinction of species, while even the superior facultative species will be driven into extinction by its overstrong mutualism on the next one; (iii) Initial population density plays a role in the coexistence of species. It is also shown that when there exists weak mutualism, an obligate species can survive by providing more benefit to the next one, and the inferior facultative species will not be driven into extinction if it can strengthen its mutualism on the next species. Moreover, Hopf bifurcation, saddle-node bifurcation and bifurcation of heteroclinic cycles are shown in the system. Projection method is extended to exhibit bistability in the three-dimensional model: when saddle-node bifurcation occurs, stable manifold of the saddle-node point divides int $R_{+}^{3}$ into two basins of attraction of two equilibria. Furthermore, Lyapunov method is applied to
\end{abstract}

*Corresponding author. E-mail address: wuhong@mail.sysu.edu.cn 
exhibit unstability of heteroclinic cycles. Numerical simulations confirm and extend our results.

Keywords. Cooperation; competition; persistence; Hopf bifurcation; saddle-node bifurcation; heteroclinic cycles

\section{Introduction}

Mutualism between species is often regarded as the interaction in which the increase of one species is beneficial to the growth of the other. However, in many situations, mutualism is density-dependant. For example, Mullerian mimics are mutualistic through facilitating the training of predators in recognizing unpalatable prey, while they are competitive since they share resources (Gilbert, 1983). Many bacterial species survive in a mutualistic association by consuming products of another one, while there is spatial competition between them since they live in the same body (Keller et al., 2006). In a light bulb powered by bacteria, several different species of microorganisms are contained in a jar, which form a closed ecosystem of synthetic biology (Zaiken et al., 2014). Each organism plays a role in the recycling of vital nutrients that each of the other microbes need to survive. Among the microorganisms, the bacteria of $E$. coli can produce light like jellyfish and the bulb could glow and recharge repeatedly. Although the microorganisms are mutualistic by recycling nutrients for others, there is spatial competition among them since they live in the same jar. Such mutualisms with negative feedbacks widely exist in natural environment (Zhang 2003, Begon et al. 2006, Agrawal et al. 2007, Holland and DeAngelis, 2010, Wang and $\mathrm{Wu}, 2011)$.

Iwata et al. (2011) established a lattice gas model to study a syntrophic association of species with spatial competition, in which the lattice consists of many sites. One empty site can be occupied by one individual of the species, and a site occupied by an individual becomes empty when the individual goes extinct (Harris, 1974; Konno, 1994). Any pair of sites on the lattice interact in a random and independent way. When an empty site is met by an individual of a species, it would be 


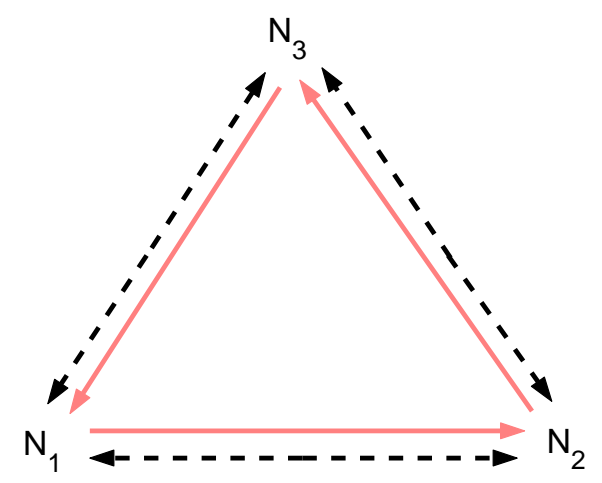

Figure 1: Mutualisms with bidirectional interactions among three species. The red and solid lines represent positive effects, while the black and dashed lines represent negative effects. The red arrow from $N_{1}$ to $N_{2}$ denotes that species 1 provides benefit (e.g., food) for species 2, so does species 2 for species 3 and species 3 for species 1 . On the other hand, the black arrow from $N_{i}$ to $N_{j}$ denotes the spatial competition between the species since they live on the same lattice.

occupied by the species if an offspring of the individual is placed on it. Thus, there exists spatial competition among different species because of limitation of sites on lattice. On the other hand, the species are mutualistic since each species provides benefit for the next one, which promotes reproduction of the species. Iwata et al. (2011) studied the lattice gas system of two species. Local stability analysis and numerical simulations demonstrate seven types of dynamics in the system. Wang and $\mathrm{Wu}$ (2015) exhibited the eighth type of dynamics by global stability analysis on an extended model. For more references, we refer to Tainaka (1989), Ruan and He (1998), Tainaka et al. (2014), Yokoi et al. (2014), Wang et al. (2015), etc. Since syntrophic associations usually consist of more than two species, extending the model of Iwata et al. (2011) to multiple species and demonstrating essential properties of the mutualisms is necessary.

In this paper, we consider a lattice gas model of three species $\mathcal{N}_{1}, \mathcal{N}_{2}$ and $\mathcal{N}_{3}$, whose population densities are represented by $N_{1}, N_{2}$ and $N_{3}$ respectively. Mutualisms between the species are cyclic as shown in Fig. 1: species 1 is beneficial to species 2 , species 2 is beneficial to species 3 , and species 3 is beneficial to species 1 in return. Thus, in our model, the three species are mutualistic in a one-directional 
loop, while there exists competition among the species.

On the lattice, a site is labeled by $\mathcal{N}_{1}$ if it is occupied by an individual of species 1. When it is empty, it is labeled by $O$. If sites $\mathcal{N}_{1}$ and $O$ interact, then the site $O$ will become $\mathcal{N}_{1}$ in a birth rate $B_{1}$. If a site is occupied by $\mathcal{N}_{1}$, then it will become $O$ in a mortality rate $m_{1}$. Thus the reactions in species 1 can be described by

$$
\begin{array}{rlrl}
\mathcal{N}_{1}+O & \rightarrow 2 \mathcal{N}_{1} & & \text { with rate } B_{1} \\
\mathcal{N}_{1} & \rightarrow O & \text { with rate } m_{1}
\end{array}
$$

where the birth rate of species 1 satisfies $B_{1}=r_{1}+a_{1} N_{3}$ (Verhulst, 1838). For simplicity, we use Type I Holling functional response $\left(a_{1} N_{3}\right)$ to describe the mutualistic effect from species 3 to 1 (Hofbauer and Sigmund, 1998). Parameter $r_{1}$ represents the intrinsic growth rate of species 1 in the absence of species 3 , and the term " $a_{1}$ " represents the mutualistic effect of an individual of species 3 on the growth of species 1. $a_{1}$ consists of two factors: $a_{1}=e_{1} s_{3}$, where $s_{3}$ represents the quantity of resource produced by an individual of species 3 and $e_{1}$ denotes the efficiency of species 1 in converting the resource into fitness. Since $m_{1}$ denotes the mortality rate of $\mathcal{N}_{1}$, we have $r_{1} \geq 0, a_{1}>0, m_{1}>0$. In the following discussions, we focus on the resource (e.g., $\left.s_{3}\right)$ produced by another species, while a similar discussion can be given for the efficiency (e.g., $e_{1}$ ).

Similarly, the reactions in species $\mathcal{N}_{2}$ can be described by

$$
\begin{aligned}
\mathcal{N}_{2}+O & \rightarrow 2 \mathcal{N}_{2} & & \text { with rate } B_{2} \\
\mathcal{N}_{2} & \rightarrow O & & \text { with rate } m_{2}
\end{aligned}
$$

where the birth rate of $\mathcal{N}_{2}$ satisfies $B_{2}=r_{2}+a_{2} N_{1}$. Parameters $r_{2}, a_{2}$ have meanings similar to those for $\mathcal{N}_{1}$. Thus, we obtain $r_{2} \geq 0, a_{2}>0, m_{2}>0$.

The reactions in species $\mathcal{N}_{3}$ can be described by

$$
\begin{aligned}
\mathcal{N}_{3}+O & \rightarrow 2 \mathcal{N}_{3} & & \text { with rate } B_{3} \\
\mathcal{N}_{3} & \rightarrow O & & \text { with rate } m_{3}
\end{aligned}
$$

where the birth rate of $\mathcal{N}_{3}$ satisfies $B_{3}=r_{3}+a_{3} N_{2}$. Parameters $r_{3}, a_{3}$ have meanings similar to those for $\mathcal{N}_{1}$. Thus, we have $r_{3} \geq 0, a_{3}>0, m_{3}>0$. 
Based on reactions in (1.1-1.3), we formed a mutualism model to characterize the lattice gas system and considered the cases of obligate mutualisms, obligatefacultative mutualisms and facultative mutualisms. In obligate mutualisms, neither species can survive alone and dynamics of the model demonstrate mechanisms by which the three species can coexist. In obligate-facultative mutualisms, at least one species cannot survive alone and dynamics of the model exhibit mechanisms by which the species can survive. In facultative mutualisms, each species can survive alone and dynamics of the model display mechanisms by which the mutualisms lead to coexistence/extinction of species.

The paper is organized as follows. The model is described in Section 2. Sections 3 and 4 exhibit stability of subsystems and equilibria. Sections 5, 6 and 7 consider obligate mutualisms, obligate-facultative mutualisms and facultative mutualisms, respectively. Discussion is in Section 8.

\section{Model}

First, we consider a lattice gas system of a single species $\mathcal{N}_{1}$. Let $N_{3}=0$ in reaction (1.1). When the number of sites on lattice is large, the "contact process" (Harris, 1974; Konno, 1994) in the system can be described by an ordinary differential equation, which is regarded as the mean-field theory of lattice model:

$$
\frac{d N_{1}}{d t}=r_{1} N_{1}\left(1-N_{1}\right)-m_{1} N_{1}
$$

where $N_{1}$ and $1-N_{1}$ are the densities of species $\mathcal{N}_{1}$ and empty sites, respectively. The first and second terms in the right-hand side come from the birth and mortality processes. The equation can be rewritten as

$$
\frac{d N_{1}}{d t}=R_{1} N_{1}\left(1-\frac{N_{1}}{K_{1}}\right), \quad R_{1}=r_{1}-m_{1}, \quad K_{1}=\frac{r_{1}-m_{1}}{r_{1}}
$$

which is a canonical logistic equation (Verhulst, 1838).

Second, we consider the lattice gas system of three species described by reactions (1.1)-(1.3). Similar to the lattice gas model of a single species, the three-species 
system can be described by

$$
\begin{aligned}
& \frac{d N_{1}}{d t}=B_{1} N_{1}\left(1-N_{1}-N_{2}-N_{3}\right)-m_{1} N_{1} \\
& \frac{d N_{2}}{d t}=B_{2} N_{2}\left(1-N_{1}-N_{2}-N_{3}\right)-m_{2} N_{2} \\
& \frac{d N_{3}}{d t}=B_{3} N_{3}\left(1-N_{1}-N_{2}-N_{3}\right)-m_{3} N_{3}
\end{aligned}
$$

where $B_{i}=r_{i}+a_{i} N_{i-1}, i=1,2,3$ and we count indices mod 3 in this work. The expression of $\left(1-N_{1}-N_{2}-N_{3}\right)$ denotes the density of empty sites, which could be assumed to satisfy $1-N_{1}-N_{2}-N_{3} \geq 0$ as shown in the proof of Theorem 2.1. Thus, on the righthand side of each equation, the first term represents the birth process of the species, while the second one denotes the mortality process.

For simplicity, we take the replacement in (2.1)

$$
\alpha_{i}=\frac{a_{i}}{m_{i}}, \quad \gamma_{i}=\frac{r_{i}}{m_{i}}, \quad i=1,2,3
$$

while we still use $a_{i}$ and $r_{i}$ to denote $\alpha_{i}$ and $\gamma_{i}$ in the following discussions. Then system (2.1) becomes

$$
\begin{aligned}
& \frac{d N_{1}}{d t}=m_{1} N_{1}\left[-1+\left(r_{1}+a_{1} N_{3}\right)\left(1-N_{1}-N_{2}-N_{3}\right)\right] \\
& \frac{d N_{2}}{d t}=m_{2} N_{2}\left[-1+\left(r_{2}+a_{2} N_{1}\right)\left(1-N_{1}-N_{2}-N_{3}\right)\right] \\
& \frac{d N_{3}}{d t}=m_{3} N_{3}\left[-1+\left(r_{3}+a_{3} N_{2}\right)\left(1-N_{1}-N_{2}-N_{3}\right)\right] .
\end{aligned}
$$

Since we are concerned with solutions of (2.2) with nonnegative initial values, we have $N_{i}(t) \geq 0$ as $t>0, i=1,2,3$. We also have the following results on the boundedness of solutions of system (2.2).

Theorem 2.1. Solutions of system (2.2) are bounded.

Proof. Assume $N_{1}+N_{2}+N_{3} \geq 1$. Then from the righthand sides of (2.2), we have $d N_{i} / d t<0, i=1,2,3$. Thus, $\lim _{\sup _{t \rightarrow \infty}} N_{1}(t)+N_{2}(t)+N_{3}(t) \leq 1$, which implies that solutions of system (2.2) are bounded.

Theorem 2.1 exhibits that orbits of (2.2) will finally satisfy $N_{1}(t)+N_{2}(t)+N_{3}(t) \leq$ 1. Thus, in the following discussions, we focus on the region

$$
\Omega=\left\{\left(N_{1}, N_{2}, N_{3}\right) \in R_{+}^{3}: N_{1}+N_{2}+N_{3} \leq 1\right\} .
$$




\section{Subsystems}

In this section, we demonstrate dynamics of subsystems in (2.2). We focus on the subsystem consisting of species 1 and 2, while similar results are given for other subsystems in Corollary 3.3.

Let $N_{3}=0$. Then system $(2.2)$ becomes the $\left(N_{1}, N_{2}\right)$-subsystem

$$
\begin{aligned}
& \frac{d N_{1}}{d t}=m_{1} N_{1}\left[-1+r_{1}\left(1-N_{1}-N_{2}\right)\right] \\
& \frac{d N_{2}}{d t}=m_{2} N_{2}\left[-1+\left(r_{2}+a_{2} N_{1}\right)\left(1-N_{1}-N_{2}\right)\right] .
\end{aligned}
$$

It follows from Theorem 2.1 that solutions of (3.1) are bounded. The following result exhibits nonexistence of periodic solution of (3.1).

Theorem 3.1. System (3.1) admits no periodic solution.

Proof. Let $P$ and $Q$ be the righthand sides of (3.1), respectively. Let $D=1 /\left(N_{1} N_{2}\right)$, which is defined in the interior of the first quadrant $\operatorname{int} R_{+}^{2}$. Then we have

$$
\frac{\partial(D P)}{\partial N_{1}}+\frac{\partial(D Q)}{\partial N_{2}}=-\frac{m_{1} r_{1}}{N_{2}}-\frac{m_{2}\left(r_{2}+a_{2} N_{1}\right)}{N_{1}}<0 .
$$

It follows from Bendixson-Dulac Theorem that (3.1) has no periodic solution.

The Jacobian matrix of (3.1) is

$$
J=\left(\begin{array}{cc}
m_{1}\left[-1+\left(1-2 N_{1}-N_{2}\right) r_{1}\right] & -m_{1} r_{1} N_{1} \\
m_{2} N_{2}\left[a_{2}\left(1-2 N_{1}-N_{2}\right)-r_{2}\right] & m_{2}\left[-1+\left(r_{2}+a_{2} N_{1}\right)\left(1-N_{1}-2 N_{2}\right)\right]
\end{array}\right) .
$$

Local stability of equilibria of (3.1) can be determined by eigenvalues of $J$, which is shown as follows.

(a) $E_{0}=(0,0)$. The trivial equilibrium $E_{0}$ always exists with eigenvalues

$$
\mu_{0}^{(1)}=m_{1}\left(r_{1}-1\right), \quad \mu_{0}^{(2)}=m_{2}\left(r_{2}-1\right)
$$

(b) $E_{1}=\left(1-\frac{1}{r_{1}}, 0\right)$. The semi-trivial equilibrium $E_{1}$ exists if $r_{1}>1$, in which it has eigenvalues

$$
\mu_{1}^{(1)}=m_{1}\left(1-r_{1}\right), \quad \mu_{1}^{(2)}=\frac{m_{2}}{r_{1}}\left[r_{2}-r_{1}+a_{2}\left(1-\frac{1}{r_{1}}\right)\right] .
$$


(c) $E_{2}=\left(0,1-\frac{1}{r_{2}}\right)$. The semi-trivial equilibrium $E_{2}$ exists if $r_{2}>1$, in which it has eigenvalues

$$
\mu_{2}^{(1)}=m_{1}\left(1-r_{2}\right), \quad \mu_{2}^{(2)}=\frac{m_{2}\left(r_{2}-r_{1}\right)}{r_{2}} .
$$

(d) $E_{12}\left(\bar{N}_{1}, \bar{N}_{2}\right)$. The positive equilibrium $E_{12}$ exists if $\bar{N}_{1}>0$ and $\bar{N}_{2}>0$ where

$$
\bar{N}_{1}=\frac{r_{1}-r_{2}}{a_{2}}, \quad \bar{N}_{2}=1-\frac{1}{r_{1}}-\frac{r_{1}-r_{2}}{a_{2}} .
$$

The Jacobian matrix of (3.1) at $E_{12}$ is

$$
J_{12}=\left(\begin{array}{cc}
-m_{1} r_{1} \bar{N}_{1} & -m_{1} r_{1} \bar{N}_{1} \\
\left(\frac{a_{2}}{r_{1}}-r_{1}\right) m_{2} \bar{N}_{2} & -m_{2} r_{1} \bar{N}_{2}
\end{array}\right)
$$

which means that

$$
\operatorname{trace} J_{12}=-m_{1} r_{1} \bar{N}_{1}-m_{2} r_{2} \bar{N}_{2}, \quad \operatorname{det} J_{12}=m_{1} m_{2} a_{2} \bar{N}_{1} \bar{N}_{2}
$$

Global dynamics of (3.1) are determined by relative positions of its isoclines.

Denote

$$
\begin{array}{ll}
l_{1}: & 1-N_{1}-N_{2}=\frac{1}{r_{1}} \\
l_{2}: & \left(r_{2}+a_{2} N_{1}\right)\left(1-N_{1}-N_{2}\right)=1 .
\end{array}
$$

Thus, $l_{1}$ is a straight line and $l_{2}$ is a hyperbola with asymptotes

$$
1-N_{1}-N_{2}=0, \quad N_{1}=-\frac{r_{2}}{a_{2}} .
$$

The expression of $l_{2}$ can be written as

$$
N_{2}\left(a_{2}, N_{1}\right)=1-N_{1}-\frac{1}{r_{2}+a_{2} N_{1}}
$$

then we have

$$
\frac{\partial^{2} N_{2}\left(a_{2}, N_{1}\right)}{\partial N_{1}^{2}}=\frac{-2 a_{2}^{2}}{\left(r_{2}+a_{2} N_{1}\right)^{3}}<0
$$

which implies that the function $N_{2}=N_{2}\left(a_{2}, N_{1}\right)$ is convex upward as shown in Fig. $2 \mathrm{a}$.

Dynamics of (3.1) are shown in the following theorem.

Theorem 3.2. Consider the $\left(N_{1}, N_{2}\right)$-subsystem (3.1). 
(i) Assume $r_{1} \leq 1$. If $r_{2}>1$, equilibrium $E_{2}$ of (3.1) is globally asymptotically stable. Otherwise, all solutions of (3.1) converge to $E_{0}$.

(ii) Assume $r_{1}>1, r_{2} \leq 1$. If $\mu_{1}^{(2)}>0$, equilibrium $E_{12}$ is globally asymptotically stable. Otherwise, all solutions of (3.1) converge to $E_{1}$, as shown in Fig. 2a.

(iii) Assume $r_{1}>1, r_{2}>1$. If $r_{1} \leq r_{2}$, all solutions of (3.1) converge to equilibrium $E_{2}$, as shown in Fig. 2b. If $r_{1}>r_{2}$ and $\mu_{1}^{(2)}>0$, equilibrium $E_{12}$ is globally asymptotically stable, as shown in Fig. 2c. If $r_{1}>r_{2}$ and $\mu_{1}^{(2)} \leq 0$, all solutions of (3.1) converge to $E_{1}$, as shown in Fig. 2d.

Proof. (i) Since $r_{1} \leq 1$, we have $d N_{1} / d t<0$ by the first equation of (3.1), which implies that $\lim _{t \rightarrow \infty} N_{1}(t)=0$. Thus, if $r_{2}>1$, we have $\lim _{t \rightarrow \infty} N_{2}(t)=1-\frac{1}{r_{2}}$ by the second equation of (3.1). If $r_{2} \leq 1$, we have $\lim _{t \rightarrow \infty} N_{2}(t)=0$.

(ii) Since $r_{2} \leq 1<r_{1}, E_{2}$ is not in the first quadrant and we obtain

$$
\mu_{0}^{(1)}>0, \quad \mu_{0}^{(2)} \leq 0, \quad \mu_{1}^{(1)}<0 .
$$

Thus $E_{0}$ is a saddle point. Indeed, assume $\mu_{0}^{(2)}=0$. Let $V(N)=\log N_{1}$. Then

$$
\left.\frac{d V}{d t}\right|_{(3.1), E_{0}}=m_{1}\left(r_{1}-1\right)=\mu_{0}^{(1)}>0
$$

which implies that $N_{1}(t)$ increases exponentially in a small neighborhood of $E_{0}$. Thus, $E_{0}$ cannot be the $\omega$-limit point of any solution of (3.1) in $\operatorname{int} R_{+}^{2}$ by the Lyapunov Theorem (Hofbauer and Sigmund, 1998). Since the positive $N_{2}$-axis is a stable manifold of $E_{0}, E_{0}$ is a saddle point.

If $\mu_{1}^{(2)}>0$, then $E_{1}$ is a saddle point. It follows from Theorem 3.1 that all solutions of (3.1) converge to equilibria. Since equilibria $E_{0}$ and $E_{1}$ are unstable, $E_{12}$ is globally asymptotically stable. If $\mu_{1}^{(2)} \leq 0$, then $\bar{N}_{2} \leq 0$ and there is no positive equilibrium of (3.1). Since $E_{0}$ is unstable, $E_{1}$ is globally asymptotically stable by Theorem 3.1, as shown in Fig. 2a.

(iii) Since $r_{1}>1$ and $r_{2}>1$, we obtain

$$
\mu_{0}^{(1)}>0, \quad \mu_{0}^{(2)}>0, \quad \mu_{1}^{(1)}<0, \quad \mu_{2}^{(1)}<0
$$

which implies that $E_{0}$ is an unstable node. 
If $r_{1} \leq r_{2}$, then $\bar{N}_{1} \leq 0$ and there is no positive equilibrium of (3.1). Since $\mu_{1}^{(1)}<0, \mu_{1}^{(2)}>0$, equilibrium $E_{2}$ is a saddle point. It follows from boundedness of solutions of (3.1) that $E_{2}$ is globally asymptotically stable, as shown in Fig. 2b.

Assume $r_{1}>r_{2}$. If $\mu_{1}^{(2)}>0$, then $E_{12}$ is globally asymptotically stable by a proof similar to the case of $r_{2} \leq 1<r_{1}$, as shown in Fig. 2c. If $\mu_{1}^{(2)} \leq 0$, then $\bar{N}_{2} \leq 0$ and there is no positive equilibrium of (3.1). $E_{1}$ is globally asymptotically stable by a proof similar to the case of $r_{2} \leq 1<r_{1}$, as shown in Fig. 2 d.

We consider other subsystems as follows. Let $N_{1}=0$. Then system (2.2) becomes the $\left(N_{2}, N_{3}\right)$-subsystem

$$
\begin{aligned}
& \frac{d N_{2}}{d t}=m_{2} N_{2}\left[-1+r_{2}\left(1-N_{2}-N_{3}\right)\right] \\
& \frac{d N_{3}}{d t}=m_{3} N_{3}\left[-1+\left(r_{3}+a_{3} N_{2}\right)\left(1-N_{2}-N_{3}\right)\right] .
\end{aligned}
$$

Let $N_{2}=0$. Then system $(2.2)$ becomes the $\left(N_{3}, N_{1}\right)$-subsystem

$$
\begin{aligned}
& \frac{d N_{3}}{d t}=m_{3} N_{3}\left[-1+r_{3}\left(1-N_{3}-N_{1}\right)\right] \\
& \frac{d N_{1}}{d t}=m_{1} N_{1}\left[-1+\left(r_{1}+a_{1} N_{2}\right)\left(1-N_{3}-N_{1}\right)\right] .
\end{aligned}
$$

Denote

$$
\begin{gathered}
\mu_{j}^{(j+1)}=\frac{m_{j+1}}{r_{j}}\left[r_{j+1}-r_{j}+a_{j+1}\left(1-\frac{1}{r_{j}}\right)\right], \quad E_{j}=\left(1-\frac{1}{r_{j}}, 0\right), \quad E_{j+1}=\left(0,1-\frac{1}{r_{j+1}}\right) \\
E_{j, j+1}\left(\bar{N}_{j}, \bar{N}_{j+1}\right) \quad \text { with } \quad \bar{N}_{j}=\frac{r_{j}-r_{j+1}}{a_{j+1}}, \quad \bar{N}_{j+1}=1-\frac{1}{r_{j}}-\frac{r_{j}-r_{j+1}}{a_{j+1}}, \quad j=2,3 .
\end{gathered}
$$

Dynamics of (3.2) and (3.3) are given in the following corollary, which is obtained from Theorem 3.2.

Corollary 3.3. Consider the subsystem (3.j), $j=2,3$.

(i) Assume $r_{j} \leq 1$. If $r_{j+1}>1$, equilibrium $E_{j+1}$ of (3.j) is globally asymptotically stable. Otherwise, all solutions of (3.j) converge to $E_{0}$.

(ii) Assume $r_{j}>1, r_{j+1} \leq 1$. If $\mu_{j}^{(j+1)}>0$, equilibrium $E_{j, j+1}$ is globally asymptotically stable. Otherwise, all solutions of (3.j) converge to $E_{j}$. 
(iii) Assume $r_{j}>1, r_{j+1}>1$.

(iiia) If $r_{j} \leq r_{j+1}$, all solutions of (3.j) converge to equilibrium $E_{j+1}$.

(iiib) Suppose $r_{j}>r_{j+1}$. If $\mu_{j}^{(j+1)}>0$, equilibrium $E_{j, j+1}$ is globally asymptotically stable. Otherwise, all solutions of (3.j) converge to $E_{j}$.

Remark 3.4. The subsystem (3.1) is different from the two-species system studied by Wang and $\mathrm{Wu}$ (2015). First, the model is different. While each species provides benefit for the other in their model, species 2 does not provide benefit for species 1 in system (3.1). Second, the result is different. For example, while obligate species can coexist in the system studied by Wang and Wu (2015), they will go extinct by Theorem 3.2(i) in system (3.1).

\section{Equilibrium}

In this section, we are concerned about equilibria of system (2.2). First, the Jacobian matrix of system (2.2) takes the form

$$
A=\left(\begin{array}{ccc}
a_{11} & a_{12} & a_{13} \\
a_{21} & a_{22} & a_{23} \\
a_{31} & a_{32} & a_{33}
\end{array}\right)
$$

where

$$
\begin{gathered}
a_{11}=m_{1}\left[-1+\left(r_{1}+a_{1} N_{3}\right)\left(1-2 N_{1}-N_{2}-N_{3}\right)\right], a_{12}=-m_{1} N_{1}\left(r_{1}+a_{1} N_{3}\right) \\
a_{13}=m_{1} N_{1}\left[-r_{1}+a_{1}\left(1-N_{1}-N_{2}-2 N_{3}\right)\right], a_{21}=m_{2} N_{2}\left[-r_{2}+a_{2}\left(1-2 N_{1}-N_{2}-N_{3}\right)\right] \\
a_{22}=m_{2}\left[-1+\left(r_{2}+a_{2} N_{1}\right)\left(1-N_{1}-2 N_{2}-N_{3}\right)\right], a_{23}=-m_{2} N_{2}\left(r_{2}+a_{2} N_{1}\right), a_{31}=-N_{3}\left(r_{3}+a_{3} N_{2}\right) \\
a_{32}=m_{3} N_{3}\left[-r_{3}+a_{3}\left(1-N_{1}-2 N_{2}-N_{3}\right)\right], a_{33}=m_{3}\left[-1+\left(r_{3}+a_{3} N_{2}\right)\left(1-N_{1}-N_{2}-2 N_{3}\right)\right] .
\end{gathered}
$$

We now consider the equilibria and their eigenvalues of the corresponding Jacobian matrices. Let $\lambda_{i}^{(j)}$ denotes the the eigenvalue of equilibrium $P_{i}$ in the $N_{j}$-axis direction. 
(a) $O(0,0,0)$. The trivial equilibrium $O$ has eigenvalues

$$
\lambda_{0}^{(1)}=m_{1}\left(r_{1}-1\right), \quad \lambda_{0}^{(2)}=m_{2}\left(r_{2}-1\right), \quad \lambda_{0}^{(3)}=m_{3}\left(r_{3}-1\right) .
$$

(b) $P_{1}\left(1-\frac{1}{r_{1}}, 0,0\right)$. The semi-trivial equilibrium $P_{1}$ has eigenvalues

$$
\lambda_{1}^{(1)}=m_{1}\left(1-r_{1}\right), \quad \lambda_{1}^{(2)}=\frac{m_{2}}{r_{1}}\left[r_{2}-r_{1}+\frac{a_{2}\left(r_{1}-1\right)}{r_{1}}\right], \quad \lambda_{1}^{(3)}=m_{3}\left(\frac{r_{3}}{r_{1}}-1\right) .
$$

Similarly, equilibrium $P_{2}\left(0,1-\frac{1}{r_{2}}, 0\right)$ has eigenvalues

$$
\lambda_{2}^{(1)}=m_{1}\left(\frac{r_{1}}{r_{2}}-1\right), \quad \lambda_{2}^{(2)}=m_{2}\left(1-r_{2}\right), \quad \lambda_{2}^{(3)}=\frac{m_{3}}{r_{2}}\left[r_{3}-r_{2}+\frac{a_{3}\left(r_{2}-1\right)}{r_{2}}\right] .
$$

Equilibrium $P_{3}\left(0,0,1-\frac{1}{r_{3}}\right)$ has eigenvalues

$$
\lambda_{3}^{(1)}=\frac{m_{1}}{r_{3}}\left[r_{1}-r_{3}+\frac{a_{1}\left(r_{3}-1\right)}{r_{3}}\right], \quad \lambda_{3}^{(2)}=m_{2}\left(\frac{r_{2}}{r_{3}}-1\right), \quad \lambda_{3}^{(3)}=m_{3}\left(1-r_{3}\right) .
$$

(c) $P_{12}\left(\bar{N}_{1}, \bar{N}_{2}, 0\right)$. When equilibrium $P_{12}$ exists in the first quadrant, it is globally asymptotically stable on the $\left(N_{1}, N_{2}\right)$-plane by Theorem 3.2 . Thus, we are concerned with its eigenvalue in the $N_{3}$-axis direction

$$
\lambda_{12}^{(3)}=\frac{m_{3}}{r_{1}}\left(r_{3}-r_{1}+a_{1} \bar{N}_{2}\right) \text { with } \quad \bar{N}_{2}=1-\frac{1}{r_{1}}-\frac{r_{1}-r_{2}}{a_{2}} .
$$

Similarly, equilibrium $P_{23}\left(0, \hat{N}_{2}, \hat{N}_{3}\right)$ has an eigenvalue in the $N_{1}$-axis direction

$$
\lambda_{23}^{(1)}=\frac{m_{1}}{r_{2}}\left(r_{1}-r_{2}+a_{2} \hat{N}_{3}\right) \text { with } \hat{N}_{3}=1-\frac{1}{r_{2}}-\frac{r_{2}-r_{3}}{a_{3}} .
$$

Equilibrium $P_{31}\left(\tilde{N}_{1}, 0, \tilde{N}_{3}\right)$ has an eigenvalue in the $N_{2}$-axis direction

$$
\lambda_{31}^{(2)}=\frac{m_{2}}{r_{3}}\left(r_{2}-r_{3}+a_{3} \tilde{N}_{1}\right) \text { with } \quad \tilde{N}_{1}=1-\frac{1}{r_{3}}-\frac{r_{3}-r_{1}}{a_{1}} .
$$

(d) $P^{*}\left(N_{1}, N_{2}, N_{3}\right)$. The positive equilibrium $P^{*}$ can be obtained by the righthand side of system (2.2). A direct computation shows that $N_{1}$ satisfies

$$
a_{2} M N_{1}^{2}-D_{2} N_{1}+a_{1} a_{3}-r_{2} C_{2}=0
$$

where

$$
M=a_{1} a_{2}+a_{2} a_{3}+a_{3} a_{1}, \quad C_{1}=a_{2} a_{3}+a_{2} r_{3}+a_{3} r_{2}-r_{1}\left(a_{2}+a_{3}\right)
$$




$$
\begin{gathered}
C_{2}=a_{3} a_{1}+a_{3} r_{1}+a_{1} r_{3}-r_{2}\left(a_{3}+a_{1}\right), \quad C_{3}=a_{1} a_{2}+a_{1} r_{2}+a_{2} r_{1}-r_{3}\left(a_{1}+a_{2}\right) \\
D_{i}=a_{i} C_{i}-r_{i} M, \quad i=1,2,3 .
\end{gathered}
$$

Thus we have two roots of $N_{1}$

$$
N_{1}^{-}=\frac{D_{2}-\sqrt{\Delta_{1}}}{2 a_{2} M}, \quad N_{1}^{+}=\frac{D_{2}+\sqrt{\Delta_{1}}}{2 a_{2} M}
$$

where

$$
\Delta_{i}=D_{i+1}^{2}-4 a_{i+1} M\left(a_{i} a_{i+2}-r_{i+1} C_{i+1}\right), \quad i=1,2,3 \text { and } i=i \bmod 3 .
$$

Similarly, we can obtain expressions of $N_{2}$ and $N_{3}$. Thus there are at most two positive equilibria of $(2.2)$

$$
\begin{aligned}
& P^{-}=\left(N_{1}^{-}, N_{2}^{-}, N_{3}^{-}\right)=\left(\frac{D_{2}-\sqrt{\Delta_{1}}}{2 a_{2} M}, \frac{D_{3}-\sqrt{\Delta_{2}}}{2 a_{3} M}, \frac{D_{1}-\sqrt{\Delta_{3}}}{2 a_{1} M}\right) \\
& P^{+}=\left(N_{1}^{+}, N_{2}^{+}, N_{3}^{+}\right)=\left(\frac{D_{2}+\sqrt{\Delta_{1}}}{2 a_{2} M}, \frac{D_{3}+\sqrt{\Delta_{2}}}{2 a_{3} M}, \frac{D_{1}+\sqrt{\Delta_{3}}}{2 a_{1} M}\right) .
\end{aligned}
$$

The Jacobian matrix of (2.2) at a positive equilibrium $P^{*}\left(N_{1}^{*}, N_{2}^{*}, N_{3}^{*}\right)$ is

$$
\left.A\right|_{P^{*}}=\left(\begin{array}{ccc}
\alpha_{1} & \alpha_{1} & \beta_{1} \\
\beta_{2} & \alpha_{2} & \alpha_{2} \\
\alpha_{3} & \beta_{3} & \alpha_{3}
\end{array}\right)
$$

where

$$
\begin{gathered}
\alpha_{1}=-N_{1}^{*}\left(r_{1}+a_{1} N_{3}^{*}\right), \alpha_{2}=-N_{2}^{*}\left(r_{2}+a_{2} N_{1}^{*}\right), \alpha_{3}=-N_{3}^{*}\left(r_{3}+a_{3} N_{2}^{*}\right) \\
\beta_{1}=\frac{a_{1}-\left(r_{1}+a_{1} N_{3}^{*}\right)^{2}}{r_{1}+a_{1} N_{3}^{*}} N_{1}^{*}, \beta_{2}=\frac{a_{2}-\left(r_{2}+a_{2} N_{1}^{*}\right)^{2}}{r_{2}+a_{2} N_{1}^{*}} N_{2}^{*}, \beta_{3}=\frac{a_{3}-\left(r_{3}+a_{3} N_{2}^{*}\right)^{2}}{r_{3}+a_{3} N_{2}^{*}} N_{3}^{*} .
\end{gathered}
$$

The characteristic equation of $\left.A\right|_{P^{*}}$ is

$$
\lambda^{3}+b_{1} \lambda^{2}+b_{2} \lambda+b_{3}=0
$$

where

$$
\begin{gathered}
b_{1}=-\left(\alpha_{1}+\alpha_{2}+\alpha_{3}\right)>0, b_{2}=\alpha_{1}\left(\alpha_{2}-\beta_{2}\right)+\alpha_{2}\left(\alpha_{3}-\beta_{3}\right)+\alpha_{3}\left(\alpha_{1}-\beta_{1}\right) \\
b_{3}=\alpha_{1} \alpha_{2} \beta_{3}+\alpha_{1} \alpha_{3} \beta_{2}+\alpha_{2} \alpha_{3} \beta_{1}-\beta_{1} \beta_{2} \beta_{3}-2 \alpha_{1} \alpha_{2} \alpha_{3} .
\end{gathered}
$$

Denote $\bar{\Delta}_{1}=b_{1}, \bar{\Delta}_{2}=b_{1} b_{2}-b_{3}, \bar{\Delta}_{3}=b_{3} \Delta_{2}$. Then $\bar{\Delta}_{1}>0$. From Hurwitz criterion, we conclude the following result. 
Theorem 4.1. Assume that $P^{*}$ is a positive equilibrium of system (2.2). If

$$
b_{3}>0, \quad b_{1} b_{2}-b_{3}>0
$$

then $P^{*}$ is asymptotically stable.

The result by $\mathrm{Yu}(2005)$ shows that when $\bar{\Delta}_{2}=0$, Hopf bifurcation occurs in system (2.2). Thus, we have the following result.

Theorem 4.2. Assume that $P^{*}$ is a positive equilibrium of system (2.2). If $b_{1} b_{2}=$ $b_{3}$, then Hopf bifurcation occurs at $P^{*}$ in system (2.2).

Proposition 4.3. Assume $m_{i}=m, r_{i}=r, i=1,2,3$. The $\omega$-limit set of solutions of system (2.2) consists of equilibria, while the $\alpha$-limit set consists of equilibria and infinity.

Proof. From (2.2) we have

$$
\begin{aligned}
& \frac{d}{d t}\left(\frac{N_{1}}{N_{3}}\right)=B(N) \frac{N_{1}}{N_{3}}\left(a_{1}-a_{3} \frac{N_{2}}{N_{3}}\right) \\
& \frac{d}{d t}\left(\frac{N_{2}}{N_{3}}\right)=B(N) \frac{N_{2}}{N_{3}}\left(a_{2} \frac{N_{1}}{N_{3}}-a_{3} \frac{N_{2}}{N_{3}}\right)
\end{aligned}
$$

with

$$
B(N)=\frac{m\left(1-N_{1}-N_{2}-N_{3}\right)}{N_{3}}>0, \quad\left(N_{1}, N_{2}, N_{3}\right) \in \operatorname{int} \Omega .
$$

Denote

$$
x_{1}=\frac{N_{1}}{N_{3}}, \quad x_{2}=\frac{N_{2}}{N_{3}} .
$$

From equation (4.5), we have

$$
\begin{aligned}
& \frac{d x_{1}}{d t}=B(N) x_{1}\left(a_{1}-a_{3} x_{2}\right) \\
& \frac{d x_{2}}{d t}=B(N) x_{2}\left(a_{2} x_{1}-a_{3} x_{2}\right) .
\end{aligned}
$$

Under a strictly monotonic change in time scale, system (4.6) is topologically equivalent to the following Lotka-Volterra equations (Hofbauer and Sigmund, 1998)

$$
\begin{aligned}
& \frac{d x_{1}}{d \tau}=x_{1}\left(a_{1}-a_{3} x_{2}\right) \\
& \frac{d x_{2}}{d \tau}=x_{2}\left(a_{2} x_{1}-a_{3} x_{2}\right) .
\end{aligned}
$$


Let $f$ and $g$ be the righthand sides of (4.7). Denote $D\left(x_{1}, x_{2}\right)=1 /\left(x_{1} x_{2}\right)$, which is defined in the interior of the first quadrant $\operatorname{int} R_{+}^{2}$.. Then we have

$$
\frac{\partial(D f)}{\partial x_{1}}+\frac{\partial(D g)}{\partial x_{2}}=-\frac{a_{3}}{x_{1}}<0
$$

which implies that there is no periodic solution of (4.7) by the Dulac's criterion (Hofbauer and Sigmund, 1998).

System (4.7) has two equilibria. $O(0,0)$ is unstable, while $\bar{x}\left(\bar{x}_{1}, \bar{x}_{2}\right)$ is asymptotically stable with

$$
\bar{x}_{1}=\frac{a_{1}}{a_{2}}, \quad \bar{x}_{2}=\frac{a_{1}}{a_{3}} .
$$

Thus, $\bar{x}$ is globally asymptotically stable in system (4.7).

Let $M(t)=\left(M_{1}(t), M_{2}(t), M_{3}(t)\right)$ be an orbit of (2.2) in the $\omega$-limit set of (2.2). From the global stability of $\bar{x}$ in (4.7), we have

$$
\frac{M_{1}(t)}{M_{3}(t)}=\frac{a_{1}}{a_{2}}, \frac{M_{2}(t)}{M_{3}(t)}=\frac{a_{1}}{a_{3}} .
$$

Thus we have $M_{1}(t)=a_{1} M_{3}(t) / a_{2}, M_{2}(t)=a_{1} M_{3}(t) / a_{3}$. It follows from the third equation of (2.2) that $M_{3}(t)$ satisfies

$$
\frac{d M_{3}}{d t}=m M_{3}\left\{-1+\left(r+a_{1} M_{3}\right)\left[1-\left(\frac{a_{1}}{a_{2}}+\frac{a_{1}}{a_{3}}+1\right) M_{3}\right]\right\} .
$$

Since the $\omega$-limit set of (4.8) consists of equilibria, so does the $\omega$-limit set of (2.2). Similarly, since the $\alpha$-limit set of (4.8) consists of equilibria and infinity, so does the $\alpha$-limit set of (2.2) in $\operatorname{int} R_{+}^{3}$.

\section{Obligate mutualisms}

In this section, we consider obligate mutualisms (i.e., $r_{i}<1, i=1,2,3$ ), which means that neither species can survive alone. By Theorem 4.1, the three species can coexist at equilibrium $P^{+}$if $P^{+}$is positive and asymptotically stable. Because conditions in Theorem 4.1 are difficult to check, we consider a symmetric situation of (2.2) and demonstrate characteristics of obligate mutualisms by global dynamics of the system. 
The symmetric situation is described by

$$
m_{i}=m, a_{i}=a, r_{i}=r<1, i=1,2,3 .
$$

Denote

$$
\Delta_{0}=a^{2}-6(2-r) a+9 r^{2}, \quad a^{ \pm}=3(2-r \pm 2 \sqrt{1-r}) .
$$

Thus, $\Delta_{0}>0$ if and only if $a>a^{+}$or $a<a^{-}$.

Theorem 5.1. Let (5.1) hold.

(i) Assume $a \geq a^{+}$. If $a>a^{+}$, there are two positive equilibria $P^{+}$and $P^{-} . P^{+}$ is asymptotically stable while $P^{-}$is a saddle point. If $a=a^{+}$, equilibria $P^{+}$ and $P^{-}$coincide and form a saddle-node point. The global stable manifold $S^{2}$ of $P^{-}$divides int $R_{+}^{3}$ into two regions: one is the basin of attraction of $P^{+}$, while the other is that of $O$, as shown in Fig. 3.

(ii) If $a<a^{+}$, all solutions of (2.2) converge to $O$.

Proof. It follows from (2.2) and (5.1) that the positive equilibrium $P^{*}$ has the form of $(N, N, N)$ with

$$
3 a N^{2}-(a-3 r) N+1-r=0
$$

which has two roots

$$
N^{ \pm}=\frac{a-3 r \pm \sqrt{\Delta_{0}}}{6 a} .
$$

We can see that there are positive equilibria of (2.2) if and only if $a \geq a^{+}$. Indeed, (a) when $a \geq a^{+}$, we have $\Delta_{0} \geq 0$ and $a-3 r \geq a^{+}-3 r=6[1-r+\sqrt{1-r}]>0$. Thus, $N^{+}+N^{-}=(a-3 r) /(3 a)>0$ and $N^{+} N^{-}=(1-r) /(3 a)>0$, which implies that system (2.2) has two positive equilibria. (b) When $a^{-}<a<a^{+}$, we have $\Delta_{0}<0$, which implies that system (2.2) has no positive equilibrium. (c) When $a \leq a^{-}$, we have $\Delta_{0} \geq 0$ and $a-3 r \leq a^{-}-3 r=6[1-r-\sqrt{1-r}]<0$. Thus, $N^{+}+N^{-}=(a-3 r) /(3 a)<0$ and $N^{+} N^{-}=(1-r) /(3 a)>0$, which implies that system (2.2) has no positive equilibria.

(i) Assume $a>a^{+}$. Then system (2.2) has two positive equilibria $P^{ \pm}$. We show stability of $P(N, N, N)$ by applying Theorem 4.1. From the notation in Theorem 
4.1, we have

$$
\begin{gathered}
\alpha_{i}=\alpha=-N(r+a N), \beta_{i}=\beta=-N[r+a N-a /(r+a N)] \\
b_{1}=-3 \alpha>0, \quad b_{2}=3 \alpha(\alpha-\beta), \quad b_{3}=-(\alpha-\beta)^{2}(2 \alpha+\beta) .
\end{gathered}
$$

Then we obtain

$$
\begin{gathered}
b_{1} b_{2}-b_{3}=-(\alpha-\beta)\left[6 \alpha^{2}+\left(\alpha^{2}+\alpha \beta+\beta^{2}\right)\right]>6 a \alpha^{2} /(r+a N)>0 \\
\left.b_{3}\right|_{N^{ \pm}}=-\frac{a^{2}}{18\left(r+a N^{ \pm}\right)^{3}}\left[(a+3 r)^{2}-12 a \pm(a+3 r) \sqrt{(a+3 r)^{2}-12 a}\right] .
\end{gathered}
$$

Thus we have $\left.b_{3}\right|_{N^{+}}>0$ and $\left.b_{3}\right|_{N^{-}}<0$. It follows from Hurwitz criterion that all eigenvalues of $P^{+}$have negative real parts, which implies that $P^{+}$is asymptotically stable. Let $\lambda_{i}$ be the eigenvalues of $P^{-}, i=1,2,3$. Since $\lambda_{1}+\lambda_{2}+\lambda_{3}=-b_{1}<0$ and $\lambda_{1} \lambda_{2} \lambda_{3}=-\left.b_{3}\right|_{N^{-}}>0$, two eigenvalues of $P^{-}$have negative real parts and the third eigenvalue is positive. Thus, $P^{-}$has a two-dimensional stable manifold $s^{2}$ in a small neighborhood of $P^{-}$by the Stable Manifold Theorem (Perko, 2001). By Proposition 4.3 , the $\alpha$-limit set of orbits of $(2.2)$ on $s^{2}$ consists of equilibria or infinity. Since equilibria $O$ and $P^{+}$are asymptotically stable, the $\alpha$-limit set of orbits (except $P^{-}$) on $s^{2}$ is infinity. Thus, the local stable manifold $s^{2}$ can be expanded to a global stable manifold $S^{2}$ by

$$
S^{2}=\bigcup_{t \leq 0, N(0) \in s^{2}} N(t)
$$

By Proposition 4.3, the $\omega$-limit set of orbits of (2.2) consists of equilibria. Therefore, the global stable manifold $S^{2}$ divides the first quadrant into two regions: one is the basin of attraction of $P^{+}$, while the other is that of $O$, as shown in Fig. 3.

Assume $a=a^{+}$. Then $P^{+}$and $P^{-}$coincide and form a saddle-node point $P^{-}$, which is shown as follows. Let $\mathbf{f}(N, a)$ be the righthand side of (2.2). According to the Sotomayer's Theorem (Perko, 2011), a saddle-node bifurcation occurs at $\left(P^{-}, a^{+}\right)$ if (a) the matrix $C=D \mathbf{f}\left(P^{-}, a^{+}\right)$has two eigenvalues with negative real parts and has a simple eigenvalue $\lambda=0$ with eigenvector $v$, while $C^{T}$ has an eigenvector $w$ corresponding to the eigenvalue $\lambda=0$. (b) The following conditions are satisfied

$$
w^{T} \mathbf{f}_{a}\left(P^{-}, a^{+}\right) \neq 0, \quad w^{T}\left[D^{2} \mathbf{f}\left(P^{-}, a^{+}\right)(v, v)\right] \neq 0 .
$$


A direct computation shows that

$$
C=\left(\begin{array}{lll}
c_{0} & c_{1} & c_{2} \\
c_{2} & c_{0} & c_{1} \\
c_{1} & c_{2} & c_{0}
\end{array}\right)
$$

with $c_{0}=c_{1}=-N(r+a N), c_{2}=-N[(r+a N)-a(1-3 N)]$. The matric $C$ is circulant and has eigenvalues (Hofbauer and Sigmund, 1998)

$$
\lambda_{0}=0, \quad \lambda_{1,2}=-\frac{a N(1-3 N)}{2} \pm i \frac{\sqrt{3} a(1-3 N)}{2}
$$

while $v=(1,1,1)$ and $w=(1,1,1)$ are eigenvectors of $C$ and $C^{T}$ corresponding to the eigenvalue $\lambda=0$.

Since $1-3 N=\left(a^{+}+3 r\right) / 2 a^{+}>0$, the real parts of $\lambda_{1,2}$ are negative. Direct computations show that

$$
w^{T} \mathbf{f}_{a}\left(P^{-}, a^{+}\right)=3 m N^{2}(1-3 N)>0, \quad w^{T}\left[D^{2} \mathbf{f}\left(P^{-}, a^{+}\right)(v, v)\right]=-3 m(a-3 r)<0
$$

which implies that $P^{-}$is a saddle-node point as $a=a^{+}$. Moreover, we can see that there is a center manifold $L_{0}: N_{1}=N_{2}=N_{3}$. On the straight line $L_{0}$, solutions of (2.2) above $P^{-}$converge to $P^{+}$, while those below $P^{-}$converge to $O$.

(ii) Assume $a<a^{+}$. Then system (2.2) has no positive equilibrium. By Proposition 4.3 , the $\omega$-limit set of orbits of $(2.2)$ consists of equilibria. Since $O$ is the unique nonnegative equilibrium and is asymptotically stable, all solutions of (2.2) converge to $O$.

Theorem 5.1 demonstrates basic properties of obligate mutualisms, in which each species cannot survive alone. First, mutualisms can lead to coexistence of the three species. As shown in Theorem 5.1(i), the three species with high initial densities can coexist at equilibrium $P^{+}$if the mutualistic effect between them is large $\left(a \geq a^{+}\right)$. Moreover, from (4.1) we have $\lim _{a \rightarrow \infty} N^{+}=1 / 3$, which implies that the three species would occupy almost all sites on lattice if each of the mutualistic effects is large. Thus, balanced strong mutualisms (i.e., $a_{1}, a_{2}$ and $a_{3}$ increase synchronously) are 
beneficial to expansion of the system. The biological reason is that with the strong mutualisms, each species can provide sufficient food for the next one, which leads to survival/expansion of each species in return. Since neither species can survive alone, it is the mutualism that leads to coexistence of the species.

Second, a weak mutualism can result in extinction of all species. Indeed, when a mutualistic effect is small (e.g., $a_{1}<1-r_{1}$ ), the comparison theorem (Cosner, 1996) shows that the first equation of (2.2) satisfies

$$
\frac{d N_{1}}{d t} \leq m_{1} N_{1}\left[-1+\left(r_{1}+a_{1}\right)\left(1-N_{1}-N_{2}-N_{3}\right)\right]<-m_{1} N_{1}\left(N_{1}+N_{2}+N_{3}\right)<0
$$

where $\left(N_{1}, N_{2}, N_{3}\right) \in \Omega$. Thus we have $\lim _{t \rightarrow \infty} N_{1}(t)=0$. From Theorem 3.2 we obtain $\lim _{t \rightarrow \infty} N_{i}(t)=0, i=2,3$. Thus one weak mutualism (e.g., $a_{1}$ ) can result in extinction of the three species although other mutualisms (e.g., $a_{2}$ and $a_{3}$ ) may be strong. The reason is that when the food produced by species 3 is not sufficient for survival of species 1 , species 1 goes extinct, which results in extinction of species 2 and 3 that cannot survive alone.

Finally, initial population density plays a role in the survival of species. As shown in Theorem 5.1(i), the three species with strong mutualisms can coexist at equilibrium $P^{+}$if their initial densities are high. The reason is that with the high densities, each species can produce a large amount of food for the next one, which leads to the survival of itself in return. However, when the densities are low, the amount of food for each species is not sufficient and all species go extinct.

Remark 5.2. In order to show global dynamics of (2.2), Theorem 5.1 considers a symmetric case of (5.1) and exhibits three properties of obligate mutualisms. When the condition in (5.1) is not satisfied, the properties are still valid for general cases of obligate mutualisms. We focus on the first property, while similar discussion can be given for others. Indeed, Theorem 4.1 implies that mutualisms can lead to species coexistence by showing local dynamics of (2.2): if the condition in Theorem 4.1 is satisfied, equilibrium $P^{*}$ is locally asymptotically stable, which means that the three species coexist at $P^{*}$ when their initial densities are near $P^{*}$. 


\section{Obligate-facultative mutualisms}

There are two types of obligate-facultative mutualisms: one facultative species with two obligate species (F-O-O), and two facultative species with one obligate species $(\mathrm{F}-\mathrm{F}-\mathrm{O})$.

\subsection{F-O-O mutualisms}

Without loss of generality, we assume $r_{1}>1 \geq \max \left\{r_{2}, r_{3}\right\}$, which implies that species 1 can survive alone but the others cannot.

By Theorem 3.2, equilibrium $O$ is globally asymptotically stable on the $\left(N_{2}, N_{3}\right)$ plane, while $P_{1}$ is globally asymptotically stable on the $\left(N_{3}, N_{1}\right)$-plane. When $\lambda_{1}^{(2)}>$ 0 , equilibrium $P_{12}$ exists and is globally asymptotically stable on the $\left(N_{1}, N_{2}\right)$-plane. Thus, if $\lambda_{12}^{(3)}>0$, system (2.2) is uniformly persistent by the Acyclic Theorem (Butler and Waltman 1986, Butler et al. 1986, Freedman and Moson 1990, Freedman et al. 1994). If $\lambda_{1}^{(2)} \leq 0, P_{1}$ is globally asymptotically stable on the $\left(N_{1}, N_{1}\right)$-plane, and thus is asymptotically stable in $R_{+}^{3}$; if $\lambda_{1}^{(2)}>0$ but $\lambda_{12}^{(3)}<0, P_{12}$ is asymptotically stable, which implies that system (2.2) is not persistent. Therefore, we conclude the following result.

Theorem 6.1. Assume $r_{1}>1, r_{2} \leq 1, r_{3} \leq 1$.

(i) If $\lambda_{1}^{(2)}>0$ and $\lambda_{12}^{(3)}>0$, system (2.2) is uniformly persistent.

(ii) If $\lambda_{1}^{(2)} \leq 0$, or $\lambda_{1}^{(2)}>0$ but $\lambda_{12}^{(3)}<0$, system (2.2) is not persistent.

Denote

$$
\bar{a}_{2}=\frac{r_{1}\left(r_{1}-r_{2}\right)}{r_{1}-1}, \quad \bar{a}_{3}=\frac{r_{2}\left(r_{2}-r_{3}\right)}{r_{2}-1}, \quad \hat{a}_{3}=\frac{r_{1}-r_{3}}{\bar{N}_{2}}, \quad \hat{a}_{1}=\frac{r_{2}-r_{1}}{\hat{N}_{3}}
$$

where $\bar{N}_{2}$ and $\hat{N}_{3}$ are defined in Section 4 . Then the condition of $\lambda_{1}^{(2)}>0$ can be written as $a_{2}>\bar{a}_{2}$, while $\lambda_{12}^{(3)}>0$ is $a_{3}>\hat{a}_{3}$.

Theorem 6.1 demonstrates basic properties of F-O-O mutualisms. First, mutualisms can lead to persistence of the system. In the situation of Theorem 6.1(i), 
species 2 and 3 cannot survive alone while species 1 can persist in the absence of others. System (2.2) is persistent if the mutualistic effects are large $\left(a_{2}>\bar{a}_{2}\right.$, $\left.a_{3}>\hat{a}_{3}\right)$.

Second, a weak mutualism can result in extinction of one/two species. As shown in Theorem 6.1(ii), if the mutualistic effect of species 1 on 2 is small $\left(a_{2}<\bar{a}_{2}\right)$, species 2 and 3 with low initial densities go extinct since $P_{1}$ is asymptotically stable. When $a_{2}>\bar{a}_{2}$ but $a_{3}<\hat{a}_{3}$, species 3 with low initial density will go extinct since $P_{12}$ is asymptotically stable.

Third, the obligate species can survive by strengthening its mutualism on the next one when there is weak mutualism. Indeed, while weak mutualism implies asymptotical stability of $P_{1}$ or $P_{12}$, the positive equilibrium $P^{+}$can still be asymptotically stable under the conditions of Theorem 4.1. In numerical simulations of Fig. 4 , we have $\bar{a}_{2}=2.2$. If $a_{2}=2.5$, then $\hat{a}_{3}=27.5$. Fig. 4a shows that the system persists when $a_{2}\left(=2.5>\bar{a}_{2}\right)$ and $a_{3}\left(=30>\hat{a}_{3}\right)$ are large. However, when $a_{2}(=2)$ is small, species 2 and 3 go extinct as shown in Fig. 4b. On the other hand, when $a_{2}(=2)$ is small but $a_{1}(=28)$ is large, species 2 and 3 can survive as shown in Fig. 4c. Moreover, when $a_{3}(=20)$ is small but $a_{1}(=30)$ is large, species 3 can still survive as shown in Fig. 4d. Similar simulation can show that when both of $a_{2}$ and $a_{3}$ are small but $a_{1}$ is large, species 3 can survive. Similar discussion can be given for the survival of species 2 by enhancing $a_{3}$ when $a_{1}$ is small.

Finally, initial density plays a role in coexistence of species. In the situation of Fig. 4c, since $a_{2}<\bar{a}_{2}$, both $P_{1}$ and $P^{+}$are asymptotically stable. Thus, initial densities in the basin of attraction of $P^{+}$imply coexistence of species, while those in the basin of attraction of $P_{1}$ imply extinction of species 2 and 3. A similar discussion can be given for the situation of Fig. $4 \mathrm{~d}$, where both $P_{12}$ and $P^{+}$are asymptotically stable.

\section{$6.2 \quad$ F-F-O mutualisms}

Without loss of generality, we consider two situations: $r_{3} \leq 1<r_{1} \leq r_{2}$ and $r_{3} \leq 1<r_{2}<r_{1}$, which implies that species 1 and 2 can survive alone but species 3 
cannot. When $r_{2}<r_{1}$, species 1 and 2 are called the superior and inferior facultative species, respectively.

\section{(I) The situation of $r_{3} \leq 1<r_{1} \leq r_{2}$}

By Theorem 3.2, equilibrium $P_{1}$ is globally asymptotically stable on the $\left(N_{3}, N_{1}\right)$ plane, while $P_{2}$ is globally asymptotically stable on the $\left(N_{1}, N_{2}\right)$-plane. If $\lambda_{2}^{(3)}>0$, then $P_{23}$ exists. Thus, when $\lambda_{23}^{(1)}>0$, the Acyclic Theorem implies that system (2.2) is uniformly persistent. If $\lambda_{2}^{(3)} \leq 0$, then $P_{2}$ is globally asymptotically stable on the $\left(N_{2}, N_{3}\right)$-plane and thus is asymptotically stable in $R_{+}^{3}$; if $\lambda_{2}^{(3)}>0$ but $\lambda_{23}^{(1)}<0$, then $P_{23}$ is asymptotically stable, which imply that system (2.2) is not persistent. Therefore, we conclude the following result.

Theorem 6.2. Assume $r_{3} \leq 1<r_{1} \leq r_{2}$.

(i) If $\lambda_{2}^{(3)}>0$ and $\lambda_{23}^{(1)}>0$, system (2.2) is uniformly persistent.

(ii) If $\lambda_{2}^{(3)} \leq 0$, or $\lambda_{2}^{(3)}>0$ but $\lambda_{23}^{(1)}<0$, system (2.2) is not persistent.

\section{(II) The situation of $r_{3} \leq 1<r_{2}<r_{1}$}

By Theorem 3.2, $P_{1}$ is globally asymptotically stable on the $\left(N_{3}, N_{1}\right)$-plane. If $\lambda_{1}^{(2)}>0$, then $P_{12}$ exists. If $\lambda_{2}^{(3)}>0$, then $P_{23}$ exists and we have $\lambda_{23}^{(1)}>0$ by $r_{2}<r_{1}$. If $\lambda_{2}^{(3)} \leq 0$, then $P_{2}$ is globally asymptotically stable on the $\left(N_{2}, N_{3}\right)$-plane.

Assume $\lambda_{2}^{(3)}=0$. Then no positive solution of (2.2) converges to $P_{2}$. Indeed, let $V(N)=\ln N_{1}$. Then

$$
\left.\frac{d V}{d t}\right|_{(2.2), P_{2}}=m_{1}\left(\frac{r_{1}}{r_{2}}-1\right)=\mu_{2}^{(1)}>0
$$

which implies that $N_{1}(t)$ increases exponentially in a small neighborhood of $P_{2}$. Thus, $P_{2}$ cannot be the $\omega$-limit point of any solution of $(2.2) \operatorname{in} \operatorname{int} R_{+}^{3}$ by the Lyapunov Theorem (Hofbauer and Sigmund, 1998). Since $\mu_{2}^{(1)}>0, P_{2}$ is a saddle point.

When $\lambda_{1}^{(2)}>0, \lambda_{12}^{(3)}>0$ and $\lambda_{2}^{(3)}>0$, or when $\lambda_{1}^{(2)}>0, \lambda_{12}^{(3)}>0$ and $\lambda_{2}^{(3)} \leq 0$, the Acyclic Theorem implies that system (2.2) is uniformly persistent. 
If $\lambda_{1}^{(2)} \leq 0, P_{1}$ is globally asymptotically stable on the $\left(N_{1}, N_{2}\right)$-plane and thus is asymptotically stable in $R_{+}^{3}$; if $\lambda_{1}^{(2)}>0$ but $\lambda_{12}^{(3)}<0, P_{12}$ is asymptotically stable, which imply that system (2.2) is not persistent. Therefore, we conclude the following result.

Theorem 6.3. Assume $r_{3} \leq 1<r_{2}<r_{1}$.

(i) If $\lambda_{1}^{(2)}>0$ and $\lambda_{12}^{(3)}>0$, system (2.2) is uniformly persistent.

(ii) If $\lambda_{1}^{(2)} \leq 0$, or $\lambda_{1}^{(2)}>0$ but $\lambda_{12}^{(3)}<0$, system (2.2) is not persistent.

Theorems 6.2-6.3 demonstrate basic properties of F-F-O mutualisms. Recall that by (6.1), $\lambda_{2}^{(3)}>0$ can be written as $a_{3}>\bar{a}_{3}$, while $\lambda_{23}^{(1)}>0$ is $a_{1}>\hat{a}_{1}$.

First, mutualisms can lead to persistence of the system. In the situation of Theorem 6.2(i), species 1 cannot survive in the $\left(N_{1}, N_{2}\right)$-subsystem. However, in the three-species system, species 1 can persist if the mutualistic effects are large $\left(a_{3}>\bar{a}_{3}, a_{1}>\hat{a}_{1}\right)$. A similar discussion can be given for survival of the obligate species 3. In the situation of Theorem 6.3, species 3 cannot survive alone while species 2 cannot survive in the $\left(N_{1}, N_{2}\right)$-subsystem if $a_{2} \leq \bar{a}_{2}$. Theorem $6.3(\mathrm{i})$ shows that when the mutualisms are strong $\left(a_{2}>\bar{a}_{2}, a_{3}>\hat{a}_{3}\right)$, species 2 and 3 can survive.

Second, a weak mutualism can result in extinction of species. In the situation of Theorem 6.2(ii), when $a_{3} \leq \bar{a}_{3}$, species 3 with small density goes extinct, which implies extinction of species 1 that cannot coexist with species 2 . Even when $a_{3}>\bar{a}_{3}$, species 1 with small density still goes extinct if $a_{1}<\hat{a}_{1}$. In the situation of Theorem 6.3(ii), when $a_{2} \leq \bar{a}_{2}$, species 2 with small density will go extinct, which implies extinction of species 3 that cannot survive alone. Even when $a_{2}>\bar{a}_{2}$, species 3 with small density still goes extinct if $a_{3}<\hat{a}_{3}$.

Third, the obligate species and the inferior facultative species can survive by strengthening its mutualism on the next one when there exists weak mutualism. In numerical simulations of Fig. 5 , we have $\bar{a}_{3}=2.2$. If $a_{3}=2.5$, then $\hat{a}_{1}=27.5$. Here, species 1 is the inferior facultative species and species 3 is obligate. We focus on species 1, while a similar discussion can be given for species 3. Fig. 5a shows that species 1 persists when the mutualisms $a_{3}\left(=2.5>\bar{a}_{3}\right)$ and $a_{1}\left(=30>\hat{a}_{1}\right)$ 
are strong. Fig. 5b displays that species 1 goes extinct when there exists weak mutualism $\left(a_{1}=0.01\right)$ with $a_{2}=3, a_{3}=2.5$. Fig. 5c exhibits that species 1 can survive when there exists weak mutualism $\left(a_{1}=20\right)$ with $a_{2}=28, a_{3}=2.5$. That is, species 1 can persist by enhancing its mutualism on species $2\left(a_{2}=28\right)$. Fig. $5 \mathrm{~d}$ shows that species 1 can still survive when there exists weak mutualism $\left(a_{3}=2\right)$ with $a_{2}=30, a_{1}=30$. That is, species 1 can persist by enhancing its mutualism on species $2\left(a_{2}=30\right)$.

In numerical simulations of Fig. 6 , we have $\bar{a}_{2}=0.6$. If $a_{2}=1$, then $\hat{a}_{3}=4.5$. Here, species 2 is the inferior facultative species and species 3 is obligate. We focus on species 3, while a similar discussion can be given for species 2. Fig. 6a shows that species 3 persists when the mutualisms $a_{2}\left(=1>\bar{a}_{2}\right)$ and $a_{3}\left(=5>\hat{a}_{3}\right)$ are strong. Fig. $6 \mathrm{~b}$ displays that species 2 and 3 go extinct when there exists weak mutualism ( $\left.a_{2}=0.1\right)$ with $a_{1}=1, a_{3}=5$. Fig. $6 \mathrm{c}$ exhibits that species 3 can survive when there exists weak mutualism $\left(a_{2}=0.59\right)$ with $a_{1}=4, a_{3}=5$. That is, species 3 can persist by enhancing its mutualism on species $1\left(a_{1}=4\right)$. Fig. 6 d shows that species 3 can still survive when there exists weak mutualism $\left(a_{3}=4.4\right)$ with $a_{1}=5, a_{2}=1$. That is, species 1 can persist by enhancing its mutualism on species $1\left(a_{1}=5\right)$.

Finally, initial density plays a role in coexistence of species. In the situation of Fig. 5c, since $a_{1}<\hat{a}_{1}$, both $P_{23}$ and $P^{+}$are asymptotically stable. Thus, initial densities in the basin of attraction of $P^{+}$imply coexistence of species, while those in the basin of attraction of $P_{23}$ imply extinction of species 1. A similar discussion can be given for the situation of Figs. $5 \mathrm{~d}, 6 \mathrm{c}-\mathrm{d}$, where there are bistable equilibria.

\section{Facultative mutualisms}

In this section, we consider facultative mutualisms (i.e., $r_{i}>1, i=1,2,3$ ), which means that each species can survive alone. Without loss of generality, we assume $r_{1} \geq \max \left\{r_{2}, r_{3}\right\}$. Thus, there are three situations: $r_{1}>\max \left\{r_{2}, r_{3}\right\}, r_{1}=r_{2}>r_{3}$ and $r_{1}=r_{2}=r_{3}$.

Suppose $r_{1}>r_{2}>r_{3}$. Since $r_{1}>r_{3}$, equilibrium $P_{1}$ is globally asymptotically stable on the $\left(N_{3}, N_{1}\right)$-plane by Theorem 3.2. Since $r_{1}>r_{2}$, equilibrium $P_{12}$ exists 
if $\lambda_{1}^{(2)}>0$. Since $r_{2}>r_{3}$, equilibrium $P_{23}$ exists if $\lambda_{2}^{(3)}>0$. From $r_{1}>r_{2}$, we have $\lambda_{23}^{(1)}>0$. Thus, (a) when $\lambda_{1}^{(2)}>0, \lambda_{12}^{(3)}>0$ and $\lambda_{2}^{(3)}>0$, the Acyclic Theorem implies that system (2.2) is uniformly persistent. Similarly, (b) when $\lambda_{1}^{(2)}>0$, $\lambda_{12}^{(3)}>0$ and $\lambda_{2}^{(3)}<0$, system (2.2) is also uniformly persistent. (c) Assume $\lambda_{1}^{(2)}>0$, $\lambda_{12}^{(3)}>0$ and $\lambda_{2}^{(3)}=0$. Since $\lambda_{2}^{(3)}=0$, equilibrium $P_{23}$ does not exist and $P_{2}$ is globally asymptotically stable on the $\left(N_{2}, N_{3}\right)$-plane by Theorem 3.2 . The orbit of (2.2) in $\operatorname{int} R_{+}^{3}$ will not converge to $P_{2}$ by a proof similar to that for Theorem 6.3. Hence, system (2.2) is uniformly persistent. On the other hand, if $\lambda_{1}^{(2)} \leq 0$, then $P_{1}$ is asymptotically stable; if $\lambda_{1}^{(2)}>0$ but $\lambda_{12}^{(3)}<0$, then $P_{12}$ is asymptotically stable, which imply that system (2.2) is not persistent.

Suppose $r_{1}>r_{3} \geq r_{2}$. Since $r_{1}>r_{3}$, equilibrium $P_{1}$ is globally asymptotically stable on the $\left(N_{3}, N_{1}\right)$-plane. Since $r_{3} \geq r_{2}$, equilibrium $P_{3}$ is globally asymptotically stable on the $\left(N_{2}, N_{3}\right)$-plane. Since $r_{1}>r_{2}$, equilibrium $P_{12}$ exists if $\lambda_{1}^{(2)}>0$. Thus, when $\lambda_{1}^{(2)}>0$ and $\lambda_{12}^{(3)}>0$, the Acyclic Theorem implies that system (2.2) is uniformly persistent. If $\lambda_{1}^{(2)} \leq 0$, then $P_{1}$ is asymptotically stable; if $\lambda_{1}^{(2)}>0$ but $\lambda_{12}^{(3)}<0$, then $P_{12}$ is asymptotically stable, which imply that system $(2.2)$ is not persistent. Therefore, we conclude the following result.

Theorem 7.1. Assume $r_{1}>\max \left\{r_{2}, r_{3}\right\}>1$.

(i) If $\lambda_{1}^{(2)}>0$ and $\lambda_{12}^{(3)}>0$, system (2.2) is uniformly persistent.

(ii) If $\lambda_{1}^{(2)} \leq 0$, or $\lambda_{1}^{(2)}>0$ but $\lambda_{12}^{(3)}<0$, system (2.2) is not persistent.

Suppose $r_{1}=r_{2}>r_{3}$. Since $r_{1}>r_{3}$, equilibrium $P_{1}$ is globally asymptotically stable on the $\left(N_{3}, N_{1}\right)$-plane. Since $r_{1}=r_{2}$, equilibrium $P_{2}$ is globally asymptotically stable on the $\left(N_{1}, N_{2}\right)$-plane. Thus, when $\lambda_{2}^{(3)}>0, P_{23}$ exists. From (4.2) and $r_{1}=r_{2}$, we have $\lambda_{23}^{(1)}>0$, which implies that system (2.2) is uniformly persistent. When $\lambda_{2}^{(3)} \leq 0, P_{23}$ is asymptotically stable and system $(2.2)$ is not persistent. Therefore, we conclude the following result.

Theorem 7.2. Assume $r_{1}=r_{2}>r_{3}>1$. If $\lambda_{2}^{(3)}>0$, system (2.2) is uniformly persistent. Otherwise, (2.2) is not persistent. 
Theorem 7.3. Assume $r_{i}=r>1, i=1,2,3$. Then there is a heteroclinic cycle $\Gamma$ on the boundary, which connects $P_{1}, P_{2}$ and $P_{3} . \Gamma$ is unstable and system (2.2) is uniformly persistent. If $m_{i}=m, i=1,2,3$, then all solutions of (2.2) in int $R_{+}^{3}$ converge to the positive equilibrium $P^{+}$.

Proof. Since $r_{i}=r>1$, it follows from Theorem 3.2 and Corollary 3.3 that equilibrium $P_{i}$ is globally asymptotically stable on the $\left(N_{i-1}, N_{i}\right)$-plane, $i=1,2,3$. Thus, there is a heteroclinic cycle $\Gamma$ on the boundary of $R_{+}^{3}$, which is in the form of $P_{1} \rightarrow P_{2} \rightarrow P_{3} \rightarrow P_{1}$.

The heteroclinic cycle $\Gamma$ is unstable. Indeed, let

$$
c_{1}=1, \quad c_{2}=\frac{m_{1} a_{1}}{m_{2} a_{2}}, \quad c_{3}=\frac{m_{1} a_{1}}{m_{3} a_{3}}
$$

then $c_{1} m_{1} a_{1}=c_{2} m_{2} a_{2}=c_{3} m_{3} a_{3}=a$. Denote

$$
V=\sum_{i=1}^{3} c_{i} \ln N_{i}=\ln \left(N_{1}^{c_{1}} N_{2}^{c_{2}} N_{3}^{c_{3}}\right)
$$

then

$$
\left.\left.\frac{d V}{d t}\right|_{(2.2), P_{1}}=-C+(r C+a N)(1-N)\right)\left.\right|_{P_{1}}=\frac{a(r-1)}{r^{2}}=\epsilon_{0}>0
$$

where $C=\sum_{i=1}^{3} c_{i} m_{i}, N=\sum_{i=1}^{3} N_{i}$. Thus, there exists $\delta_{1}>0$ such that when $P \in O\left(P_{1}, \delta_{1}\right) \bigcap \operatorname{int} R_{+}^{3}$, we have

$$
\left.\frac{d V}{d t}\right|_{(2.2), P}>\frac{\epsilon_{0}}{2}>0
$$

Similarly, there exists $\delta_{i}>0$ such that when $P \in O\left(P_{i}, \delta_{i}\right) \bigcap \operatorname{int} R_{+}^{3}$, we have

$$
\left.\frac{d V}{d t}\right|_{(2.2), P}>\frac{\epsilon_{0}}{2}>0, \quad i=2,3 \text {. }
$$

It follows from the continuity of solutions of (2.2) on initial values, there exists $0<\delta<\max _{1 \leq i \leq 3}\left\{\delta_{i}\right\}$ such that when $P \in O(\Gamma, \delta) \bigcap \operatorname{int} R_{+}^{3}$, we have

$$
\left.\frac{d V}{d t}\right|_{(2.2), P}>\frac{\epsilon_{0}}{4}>0
$$

which implies that $N_{1}(t)^{c_{1}} N_{2}(t)^{c_{2}} N_{3}(t)^{c_{3}}$ increases exponentially in the neighborhood $O(\Gamma, \delta) \bigcap \operatorname{int} R_{+}^{3}$. Thus, the heteroclinic cycle $\Gamma$ is repellant. 
Since $O$ is unstable, system (2.2) is uniformly persistent.

Assume $m_{i}=m, i=1,2,3$. It follows from Proposition 4.3 that all solutions of $(2.2)$ converge to equilibria. Since $P_{1}$ is globally asymptotically stable on the $\left(N_{3}, N_{1}\right)$-plane with $\lambda_{1}^{(2)}>0$, solutions of $(2.2)$ in $\operatorname{int} R_{+}^{3}$ will not converge to $P_{1}$ by a proof similar to that for Theorem 6.3. Similarly, solutions of (2.2) in $\operatorname{int} R_{+}^{3}$ will not converge to $P_{2}$ and $P_{3}$.

From the righthand side of (2.2), the positive equilibrium $P^{*}\left(N_{1}, N_{2}, N_{3}\right)$ satisfies

$$
N_{2}=\frac{a_{2}}{a_{3}} N_{1}, \quad N_{3}=\frac{a_{2}}{a_{1}} N_{1}
$$

Thus, $N_{1}$ satisfies

$$
a_{2} b N_{1}^{2}-\left(a_{2}-r b\right) N_{1}+1-r=0
$$

which has a unique positive root since $1-r<0$. Thus there is a unique positive equilibrium $P^{+}$of (2.2).

Since $O$ is hyperbolic and unstable, all solutions of $(2.2)$ in int $R_{+}^{3}$ converge to $P^{+}$.

Theorems 7.1-7.3 demonstrate basic properties of facultative mutualisms. We focus on Theorem 7.1, while similar discussion can be given for Theorems 7.2-7.3. Recall that by (6.1), $\lambda_{1}^{(2)}>0$ can be written as $a_{2}>\bar{a}_{2}$, and $\lambda_{12}^{(3)}>0$ is $a_{3}>\hat{a}_{3}$.

First, mutualisms can lead to persistence of the system. In the situation of Theorem 7.1, species 3 cannot survive in the $\left(N_{3}, N_{1}\right)$-subsystem. However, in the three-species system, species 3 can persist if the mutualistic effects are large $\left(a_{2}>\bar{a}_{2}\right.$, $\left.a_{3}>\hat{a}_{3}\right)$.

Second, a weak mutualism can result in extinction of species. In Theorem 7.1(ii), when $a_{2}<\bar{a}_{2}$, species 2 with small density goes extinct, which implies extinction of species 3 that cannot coexist with species 1 . Even when $a_{2}>\bar{a}_{2}$, species 3 with small density still goes extinct if $a_{3}<\hat{a}_{3}$.

Third, the inferior facultative species can survive by strengthening its mutualism on the next one when there exists weak mutualism. In numerical simulations of Fig. 7 , we have $\bar{a}_{2}=0.7$. If $a_{2}=1$, then $\hat{a}_{3}=3.5$. Here, species 2 and 3 are the inferior species. We focus on species 3 , while a similar discussion can be given for species 
2. Fig. 7a shows that species 3 persists when the mutualisms $a_{2}\left(=1>\bar{a}_{2}\right)$ and $a_{3}\left(=4>\hat{a}_{3}\right)$ are strong. Fig. $7 \mathrm{~b}$ displays that species 3 goes extinct when there exists weak mutualism $\left(a_{2}=0.1\right)$ with $a_{1}=1, a_{3}=4$. Fig. $7 \mathrm{c}$ exhibits that species 3 can survive when there exists weak mutualism $\left(a_{2}=0.69\right)$ with $a_{1}=5, a_{3}=4$. That is, species 3 can persist by enhancing its mutualism on species $1\left(a_{1}=5\right)$. Fig. $7 \mathrm{~d}$ shows that species 3 can still survive when there exists weak mutualism $\left(a_{3}=3.49\right)$ with $a_{1}=8, a_{2}=1$. That is, species 3 can persist by enhancing its mutualism on species $1\left(a_{1}=8\right)$.

Finally, initial density plays a role in coexistence of species. In the situation of Fig. 7c, since $a_{2}<\bar{a}_{2}$, both $P_{1}$ and $P^{+}$are asymptotically stable. Thus, initial densities in the basin of attraction of $P^{+}$imply coexistence of species, while those in the basin of attraction of $P_{1}$ imply extinction of species 2 and 3 . A similar discussion can be given for the situation of Fig. $7 \mathrm{~d}$, where both $P_{12}$ and $P^{+}$are asymptotically stable.

Remark 7.4. The bifurcation of heteroclinic cycles can occur in facultative mutualisms. Indeed, fix $r_{1}=r_{2}>1$ and let $r_{3}>1$ vary. When $r_{3}<r_{1}=r_{2}$, there is no a heteroclinic cycle and Theorem 7.2 shows that $P_{2}$ is asymptotically stable, while system (2.2) is not persistent. When $r_{3}=r_{1}=r_{2}$, a heteroclinic cycle emerges and Theorem 7.3 exhibits that system (2.2) is uniformly persistent. When $r_{3}>r_{1}=r_{2}$, the heteroclinic cycle disappears and Theorem 7.1 shows that $P_{3}$ is asymptotically stable, while system (2.2) is not persistent. Thus, when $r_{3}$ increases, the bifurcation of heteroclinic cycles occurs and dynamics of the system fluctuate between persistence and non-persistence.

\section{Discussion}

In this paper, we considered mutualisms in a three-species system where each species provides benefit to the next one in a one-directional loop, but there exists spatial competition among them. Dynamics of the model demonstrate mechanisms by which the mutualisms lead to coexistence/extinction of the species.

Periodic oscillations can occur in the system by Hopf bifurcation as shown in 
Theorem 4.2. In numerical simulations of Fig. 8a-b, the length of time-axis is 40 and the time step is 0.01. We fix $r_{1}=0.9, r_{2}=0.8, r_{3}=0.7, m_{i}=1$ and initial values $N_{i}(0)=0.3, a_{i}=a, i=1,2,3$. Since $r_{i}<1$ for $i=1,2,3$, each species cannot survive alone. Fig. 8a displays that the species coexist at an equilibrium when the mutualisms are intermediate $(a=6.5)$. Moreover, when the mutualisms are strong $(a=50)$, Hopf bifurcation occurs and the species coexist in periodic oscillations as shown in Fig. 8b. Further simulations show that when $a_{i}$ increases, the amplitude of the periodic oscillations decreases while the frequency increases. The biological meaning is: the stronger the mutualism, the more stable the species coexistence. Periodic oscillations also occur in other mutualism systems with bidirectional interactions. For example, Bachelot et al. (2015) built a multiprey species/predator model combined with a bidirectional resource exchange system. Numerical simulations exhibit that periodic oscillations appear in the model consisting of plant, natural enemy of the plant, and mycorrhizal fungi. Moreover, when the benefits of the mycorrhizal symbiosis are greater than the costs, there is a mismatch between plant and mycorrhizal fungal dynamics; when the benefits are less than the costs, plant and mycorrhizal fungal dynamics are synchronous.

Both weak mutualism and extremely strong mutualism will result in extinction of species. Since we have discussed weak mutualism in Sections 5-7, we focus on extremely strong mutualism. Indeed, from (4.4) we have $\lim _{a_{3} \rightarrow \infty} N_{2}^{+}=0$, which implies extinction of species 2 as its mutualism on species 3 is extremely strong. Thus, even the superior facultative species will be driven into extinction by its overstrong mutualism on the next one. The biological reason is that there exists spatial competition between the species. In numerical simulations of Figs. 8c-d, the length of time-axis is 40 and the time step is 0.01. We fix $r_{1}=0.9, r_{2}=0.8, r_{3}=0.7, a_{1}=$ $a_{2}=6.5, m_{i}=1, N_{i}(0)=0.3, i=1,2,3$, and let $a_{3}$ vary. Fig. $8 \mathrm{c}$ shows that all species go extinct when a mutualism is weak $\left(a_{3}=1\right)$. Fig. 8 d displays that when $a_{3}$ increases from 50, 100 to 500 , the density of species $2\left(N_{2}^{+}\right)$decreases monotonically and tends toward 0 . Thus species 2 goes extinct, which implies extinction of species 3 and 1 that cannot survive alone. Since the species can coexist in intermediate 
mutualisms as shown in Fig. 8a, it is the extremely strong/weak mutualism that leads to the extinction.

Results in this work may be helpful for understanding mutualisms in a onedirectional loop in real situations. For example, in the light bulb powered by bacteria, Theorem 5.1 shows that if the mutualisms between the bacteria are symmetric and intermediate, the closed ecosystem would persist at a steady state as shown in Fig. 8a, which implies that the bulb can produce stable light. Moreover, Theorem 4.2 and the simulation in Fig. 8b exhibits that if the mutualisms become strong, there may exist periodic solutions, which implies that light produced in bulb may be in oscillations. While this work focuses on three-species systems, considering nspecies systems may be important and interesting. We'll pursue this in a future work.

Acknowledgements: We grateful to the three anonymous reviewers for their careful reading, helpful comments and suggestions that really helped me to improve the presentation of the paper. This work was supported by NSFC (11571382) of "Bi-directional mutualisms based on pollen-transportation", NSF of Guangdong (S2012010010320), and Student Research Creation Program of Sun Yat-sen University.

\section{References}

[1] A.A. Agrawal, D.D. Ackerly, F. Adler, A.E. Arnold, C. Cáceres, D.F. Doak et al. Filling key gaps in population and community ecology. Front. Ecol. Environ. 5(2007): 145-152.

[2] B. Bachelot, M. Uriarte, and K. McGuire, Interactions among mutualism, competition, and predation foster species coexistence in diverse communities. Theoretical Ecology. In Press (2015): DOI 10.1007/s12080-015-0251-2.

[3] M. Begon, C.R. Townsend, J.L. Harper, Ecology: From Individuals to Ecosystems. Wiley, New York, 2006. 
[4] G. Butler, P. Waltman, Persistence in Dynamical Systems. Journal of Differential Equations, 63(1986): 255-263.

[5] G. Butler, H.I. Freedman, P. Waltman, Uniformly Persistent Systems. American Mathematical Society, 96(1986): 425-430.

[6] C. Cosner, Variability, vagueness and comparison methods for ecological models. Bull. Math. Biol. 58(1996): 207-246.

[7] H.I. Freedman and P. Moson, Persistence definitions and their connections, Proc. Amer. Math. Soc. 109(1990):1025-1033.

[8] H.I. Freedman, S. Ruan and M. Tang, Uniform persistence and flows near a closed positively invariant set, J. Dynam. Differential Equations 6(1994): 583600.

[9] L.E. Gilbert, Coevolution and mimicry. In Coevolution (ed. D. Futuyma and M. Slatkin), pp. 263-281. Sunderland, MA: Sinauer, 1983.

[10] Harris, T.E., Contact interaction on a lattice. Annals of Probability 2(1974): 969-988.

[11] J.N. Holland, D.L. DeAngelis, A consumer-resource approach to the densitydependent population dynamics of mutualism. Ecology 91(2010): 1286-1295.

[12] J. Hofbauer, K. Sigmund, Evolutionay Games and Population Dynamics. Cambridge University Press, Cambridge, UK, 1998.

[13] S. Iwata, K. Kobayashi, S, Higa, J. Yoshimura, K. Tainaka, A simple population theory for mutualism by the use of lattice gas model. Ecological Modelling, 222(2011): 2042-2048.

[14] L. Keller, M.G. Surette, Communication in bacteria: an ecological and evolutionary perspective. Nature Reviews Microbiology 4(2006): 249-258.

[15] Konno, N., Phase Transition of Interacting Particle Systems. World Scientific, Singapore, 1994. 
[16] S. Ruan, X. He, Global stability in chemostrt-type competition models with nutrient recycling. SIAM J. APPL. MATH. 58(1998): 170-192.

[17] L. Perko, Differential Equations and Dynamical Systems, Springer-Verlag, New York, 2001.

[18] K. Tainaka, Stationary pattern of vortices or strings in biological systems: lattice version of the Lotka-Volterra model. Phys. Rev. Lett, 63(1989): 2688-2691.

[19] K. Tainaka, T. Ushimaru, T. Hagiwara1, J. Yoshimura, Lattice Gas Model for Budding Yeast: A New Approach for Density Effects. Procedia Computer Science, 29(2014): 270-280.

[20] P.F. Verhulst, Notice sur la loi que la population suit dans son accroissement. Correspondances Mathematiques et Physiques 10(1838): 113-121.

[21] Y. Wang, H. Wu, A mutualism-competition model characterizing competitors with mutualism at low density, Mathematical and Computer Modeling, 53 (2011): 1654-1663.

[22] Y. Wang, D.L. DeAngelis and J.N. Holland. Dynamics of an ant-plant-pollinator model. Commun. Non. Sci. Nume. Simu. 20(2015):950-964.

[23] Y. Wang and H. Wu. Stability of mutualisms in a lattice gas system of two species. Electron. J. Diff. Equ., 2(2015): 1-20.

[24] H. Yokoi, T. Uehara, T. Kawai, Y. Tateoka, K. Tainaka, Lattice and lattice gas models for commensalism: two shellfishes in intertidal zone. Open Journal of Ecology, 4(2014): 671-677.

[25] P. Yu, Closed-form conditions of bifurcation points for general differential equations. International Journal of Bifurcation and Chaos. 15(2005): 1467-1483

[26] A. Zaiken, Cohn, A. Beckman, 2014. http://news.discovery.com/tech/alternativepower-sources/bacteria-powered-light-bulb-is-electricity-free-130815.htm 
[27] Z. Zhang, Mutualism or cooperation among competitors promotes coexistence and competitive ability, Ecological Modelling, 164(2003): 271-282.

(a) $r_{2}=1, a_{2}=2$

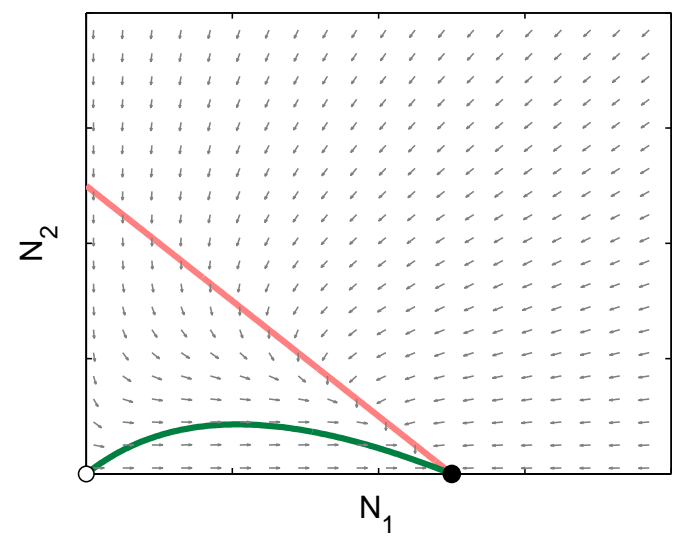

(c) $r_{2}=1.5, a_{2}=4$

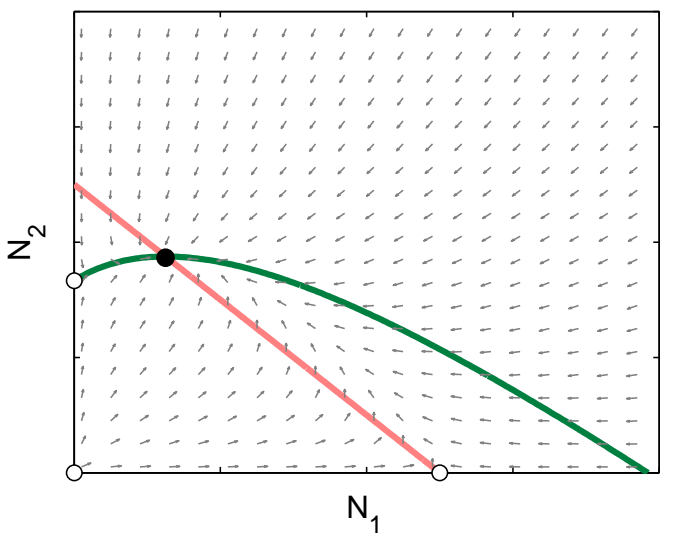

(b) $r_{2}=2, a_{2}=2$

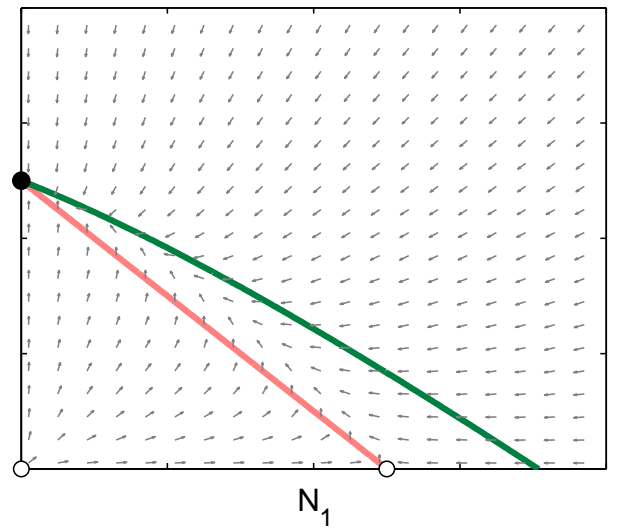

(d) $r_{2}=1.5, a_{2}=1$

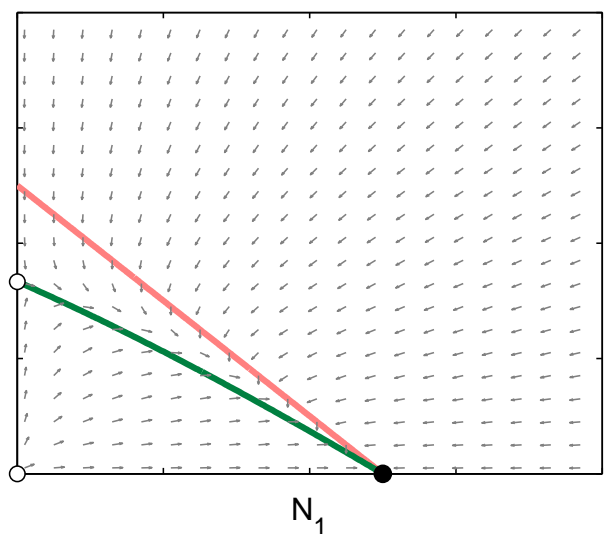

Figure 2: Dynamics of the $\left(N_{1}, N_{2}\right)$-subsystem (3.1). Fix $r_{1}=2, m_{1}=m_{2}=1$ and let $r_{2}, a_{2}$ vary. (a) When $r_{2} \leq 1$ and $a_{2}(=2)$ is small, species 2 goes extinct. (b) When $r_{2} \geq r_{1}>1$, species 1 goes extinct. (c) When $1<r_{2}<r_{1}$ and $a_{2}(=4)$ is large, the two species coexist at equilibrium $E_{12}$. (d) When $1<r_{2}<r_{1}$ and $a_{2}(=1)$ is small, species 2 goes extinct. 


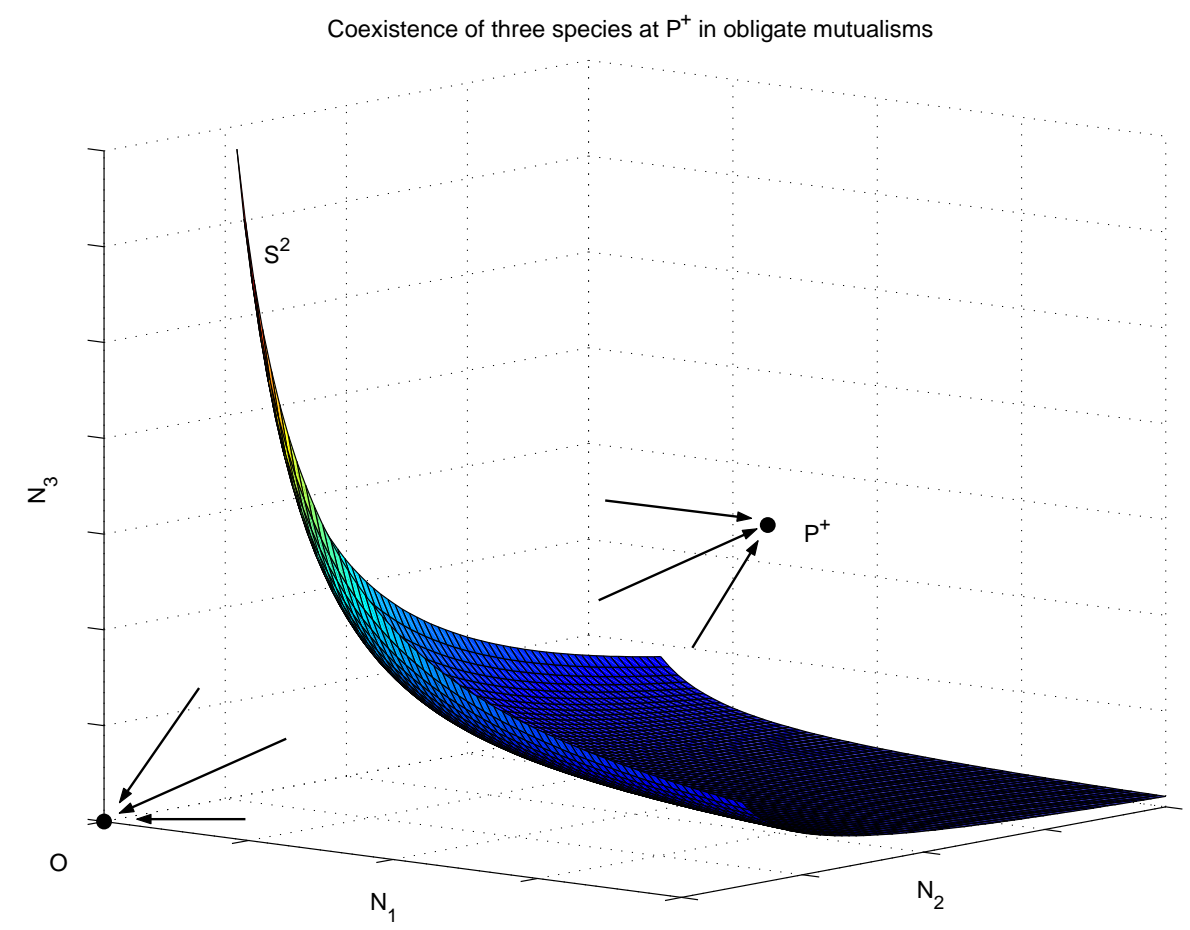

Figure 3: Dynamics of obligate mutualisms. Let $r_{i}=0.9, m_{i}=1, a_{i}=7$. There are two positive equilibria $P^{+}$and $P^{-} \cdot P^{+}$is asymptotically stable while $P^{-}$is a saddle point. The global stable manifold $S^{2}$ of $P^{-}$divides $\operatorname{int} R_{+}^{3}$ into two regions: one is the basin of attraction of $P^{+}$, while the other is that of $O$. 
(a) $a_{1}=3, a_{2}=2.5, a_{3}=30$

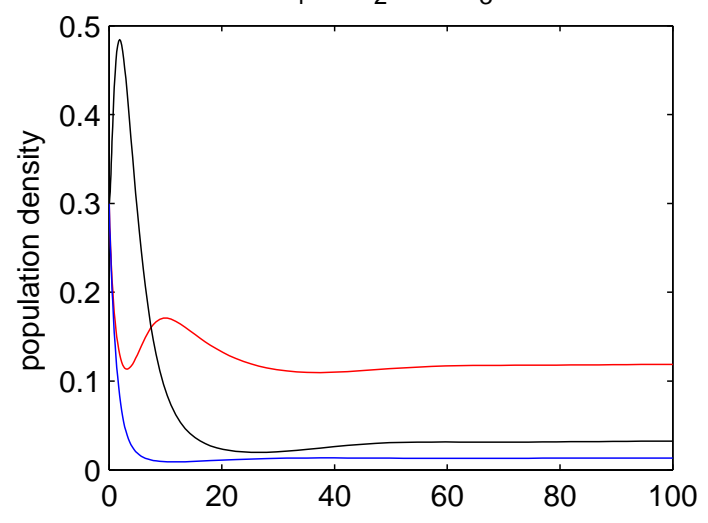

(c) $a_{1}=28, a_{2}=2.0, a_{3}=30$

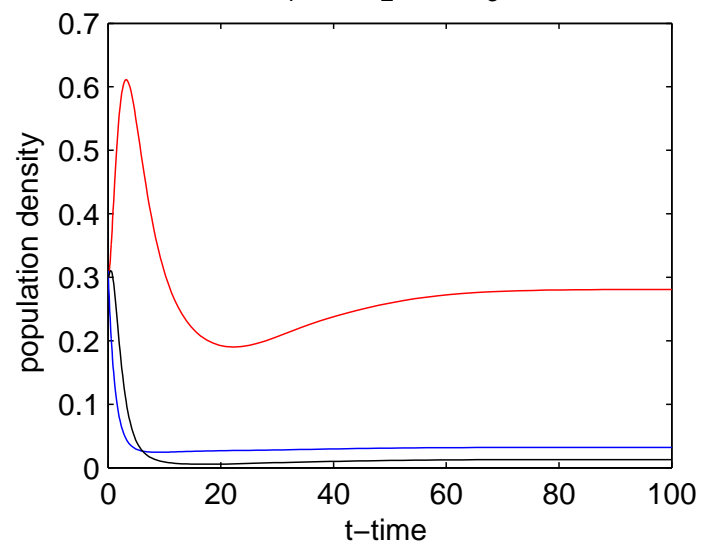

(b) $a_{1}=3, a_{2}=2.0, a_{3}=30$

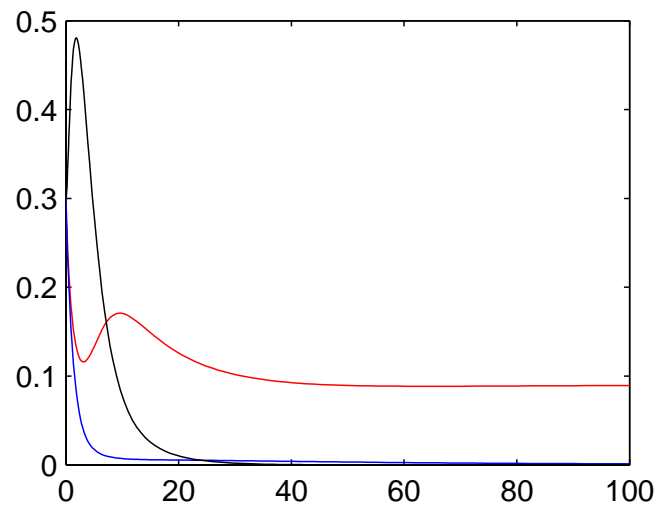

(d) $a_{1}=30, a_{2}=2.5, a_{3}=20$

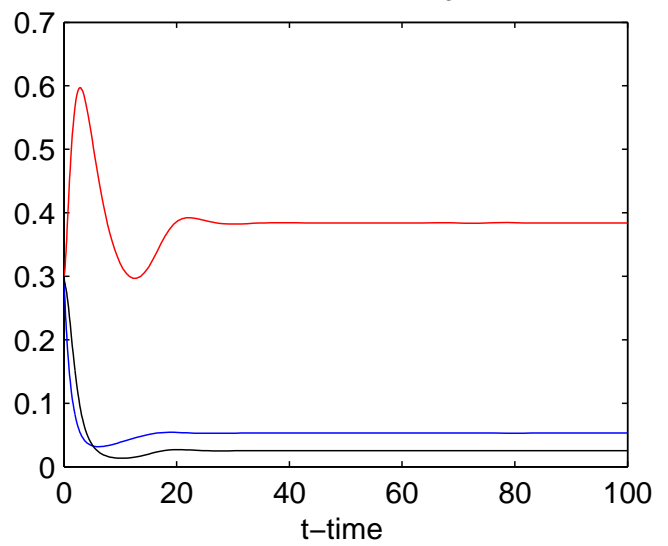

Figure 4: Solutions in F-O-O mutualisms. The red, blue and black curves represent solutions of $N_{1}(t), N_{2}(t)$ and $N_{3}(t)$, respectively. Fix $r_{1}=1.1, r_{2}=0.9, r_{3}=$ $0.8, m_{i}=1$ and initial values $N_{i}(0)=0.3, i=1,2,3$. (a) When the mutualistic effects $a_{2}(=2.5)$ and $a_{3}(=30)$ are large, the three species persist. (b) When $a_{2}(=2.0)$ and $a_{1}(=3)$ are small, species 2 and 3 go extinct while species 1 persists. (c) When $a_{2}(=2.0)$ is small but $a_{1}(=28)$ is large, the three species coexist at equilibrium $P^{+}$. (d) When $a_{2}(=2.5)$ is large but $a_{3}(=20)$ is small, species 3 can survive if $a_{1}(=30)$ is large. 
(a) $a_{1}=30, a_{2}=3, a_{3}=2.5$

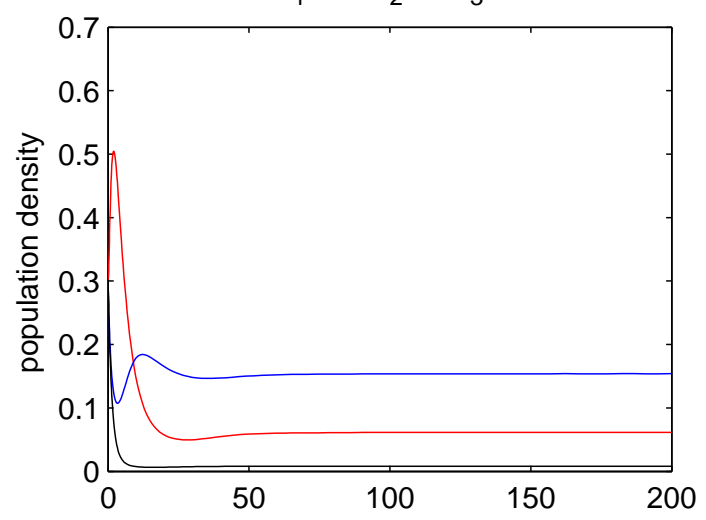

(c) $a_{1}=20, a_{2}=28, a_{3}=2.5$

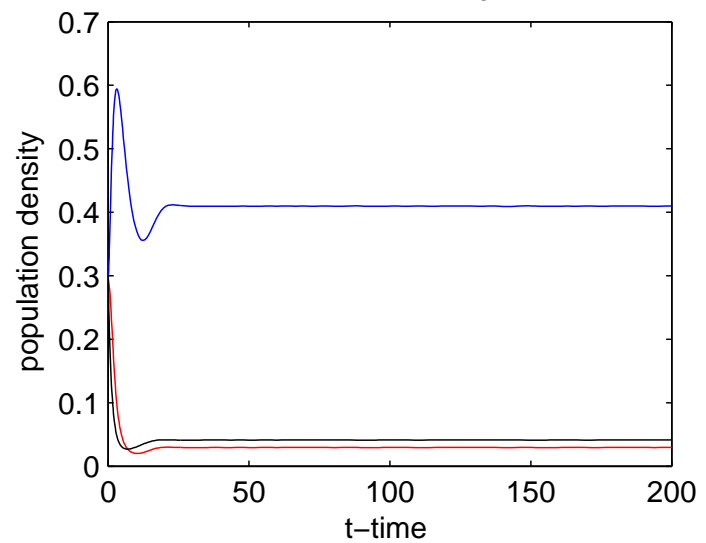

(b) $\mathrm{a}_{1}=0.01, \mathrm{a}_{2}=3, \mathrm{a}_{3}=2.5$

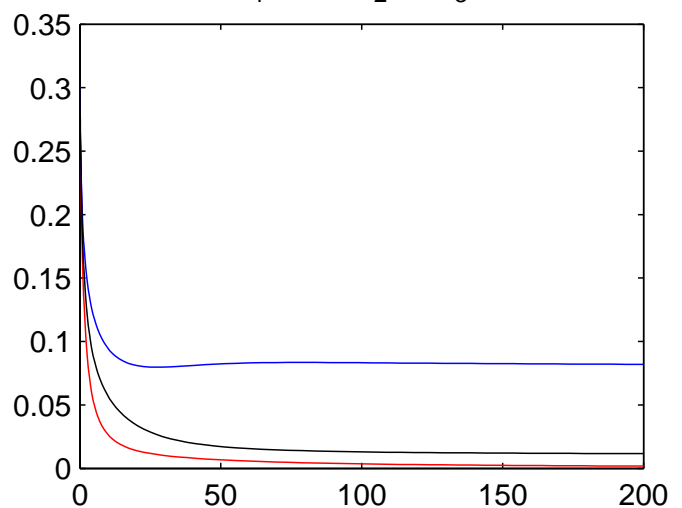

(d) $a_{1}=30, a_{2}=30, a_{3}=2$

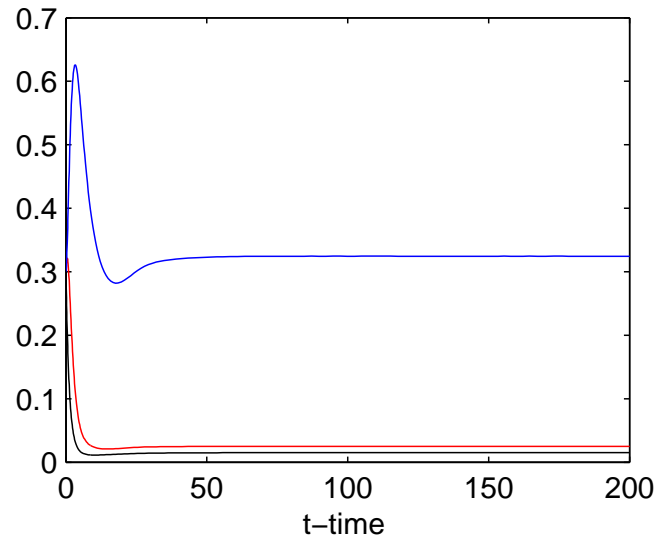

Figure 5: Solutions in F-F-O mutualisms. The red, blue and black curves represent solutions of $N_{1}(t), N_{2}(t)$ and $N_{3}(t)$, respectively. Fix $r_{1}=r_{2}=1.1, r_{3}=0.9, m_{i}=1$ and initial values $N_{i}(0)=0.3, i=1,2,3$. (a) When the mutualistic effects $a_{1}(=30)$ and $a_{3}(=2.5)$ are large, system (2.2) is persistent. (b) When $a_{1}(=0.01)$ is small with $a_{3}(=2.5)$, species 2 and 3 persist while species 1 goes extinct. (c) When $a_{1}(=20)$ is small but $a_{2}(=28)$ is large, the three species coexist at equilibrium $P^{+}$. (d) When $a_{3}(=2)$ is small but $a_{2}(=30)$ is large, species 3 can survive if $a_{1}(=30)$ is large. 
(a) $a_{1}=1, a_{2}=1, a_{3}=5$

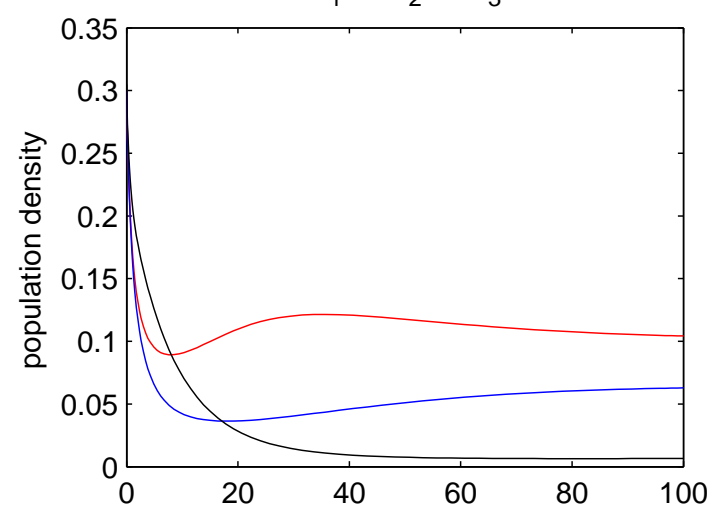

(c) $a_{1}=4, a_{2}=0.59, a_{3}=5$

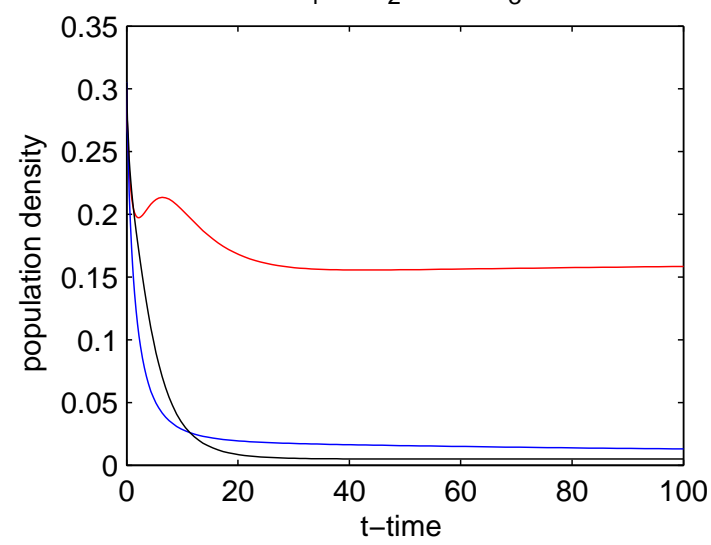

(b) $a_{1}=1, a_{2}=0.1, a_{3}=5$

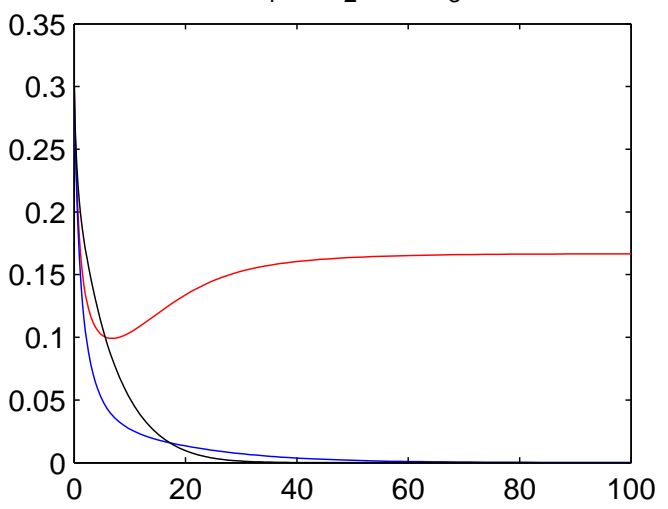

(d) $a_{1}=5, a_{2}=1, a_{3}=4.4$

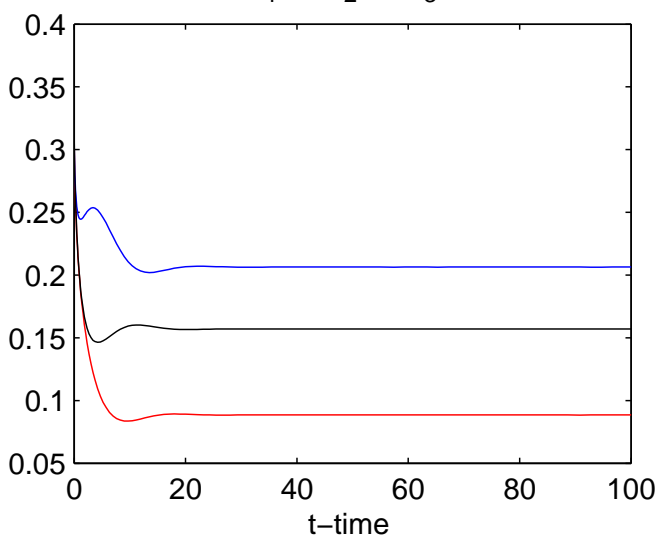

Figure 6: Solutions in F-F-O mutualisms. The red, blue and black curves represent solutions of $N_{1}(t), N_{2}(t)$ and $N_{3}(t)$, respectively. Fix $r_{1}=1.2, r_{2}=1.1, r_{3}=$ $0.9, m_{i}=1$ and initial values $N_{i}(0)=0.3, i=1,2,3$. (a) When the mutualistic effects $a_{2}(=1)$ and $a_{3}(=5)$ are large, system (2.2) is persistent. (b) When $a_{2}(=0.1)$ is small and $a_{1}(=1)$ is small, species 2 and 3 go extinct while species 1 persists. (c) When $a_{2}(=0.59)$ is small but $a_{1}(=4)$ is large, the three species coexist at equilibrium $P^{+}$. (d) When $a_{2}(=1)$ is large and $a_{3}(=4.4)$ is small, species 3 can survive if $a_{1}(=5)$ is large. 
(a) $a_{1}=1, a_{2}=1, a_{3}=4$

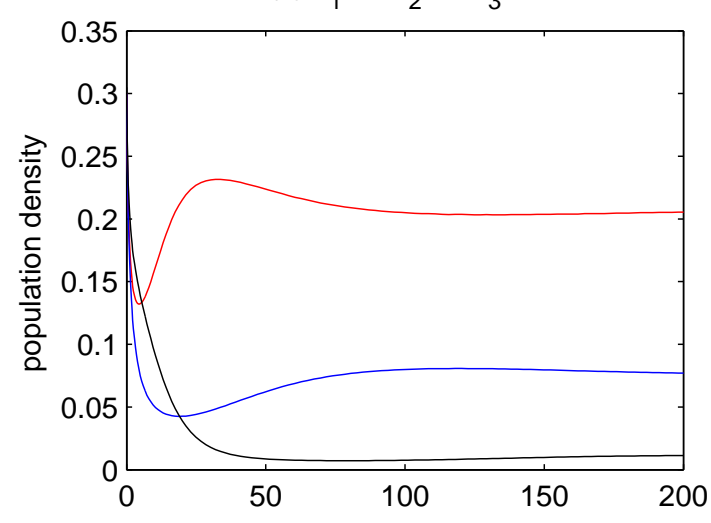

(c) $a_{1}=5, a_{2}=0.69, a_{3}=4$

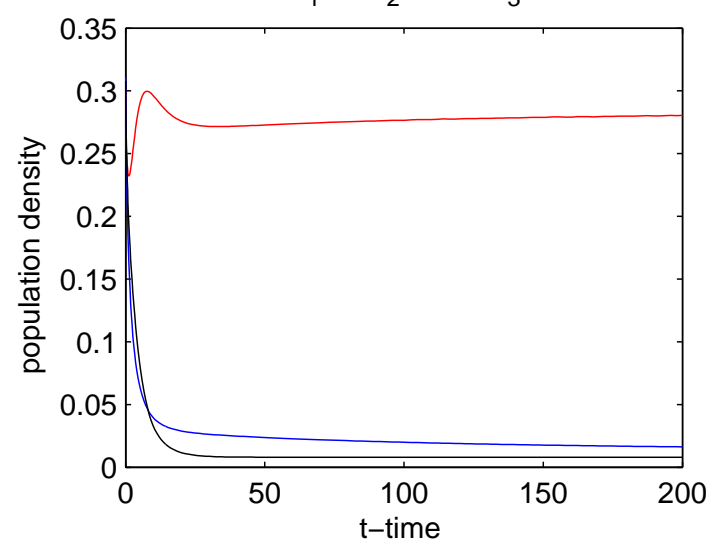

(b) $a_{1}=1, a_{2}=0.1, a_{3}=4$

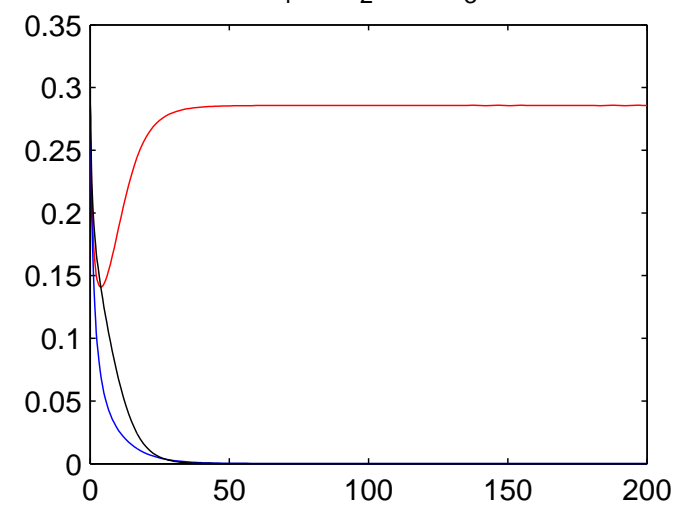

(d) $a_{1}=8, a_{2}=1, a_{3}=3.49$

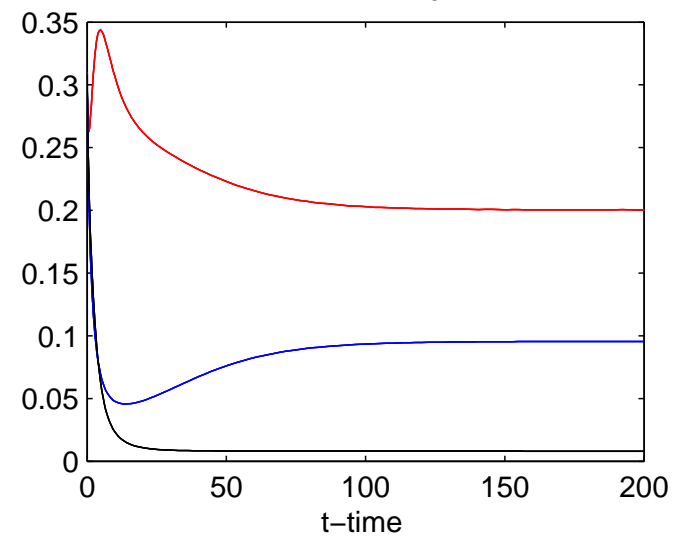

Figure 7: Solutions in facultative mutualisms. The red, blue and black curves represent solutions of $N_{1}(t), N_{2}(t)$ and $N_{3}(t)$, respectively. Fix $r_{1}=1.4, r_{2}=1.2, r_{3}=$ $1.1, m_{i}=1$ and initial values $N_{i}(0)=0.3, i=1,2,3$. (a) When the mutualistic effects $a_{2}(=1)$ and $a_{3}(=4)$ are large, system (2.2) is persistent. (b) When $a_{2}(=0.1)$ is small and $a_{1}(=1)$ is small, species 2 and 3 go extinct while species 1 persists. (c) When $a_{2}(=0.69)$ is small but $a_{1}(=5)$ is large, the three species coexist at equilibrium $P^{+}$. (d) When $a_{2}(=1)$ is large and $a_{3}(=3.49)$ is small, species 3 can survive if $a_{1}(=8)$ is large. 
(a) $\mathrm{a}_{1}=\mathrm{a}_{2}=\mathrm{a}_{3}=6.5$

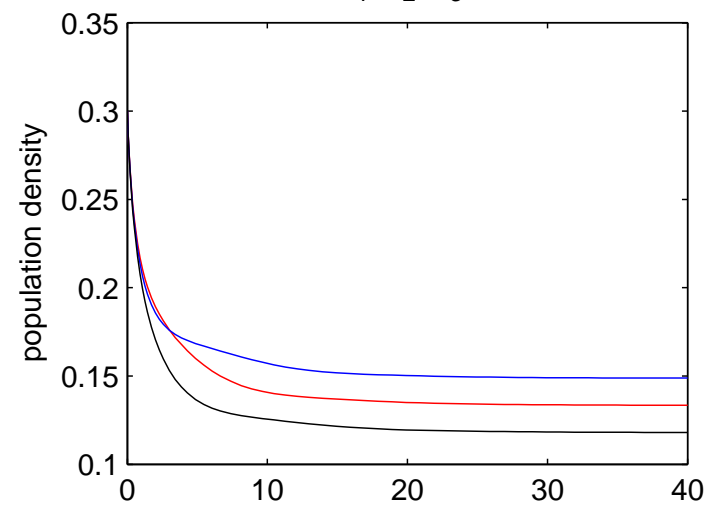

(c) $a_{1}=a_{2}=6.5, a_{3}=1$

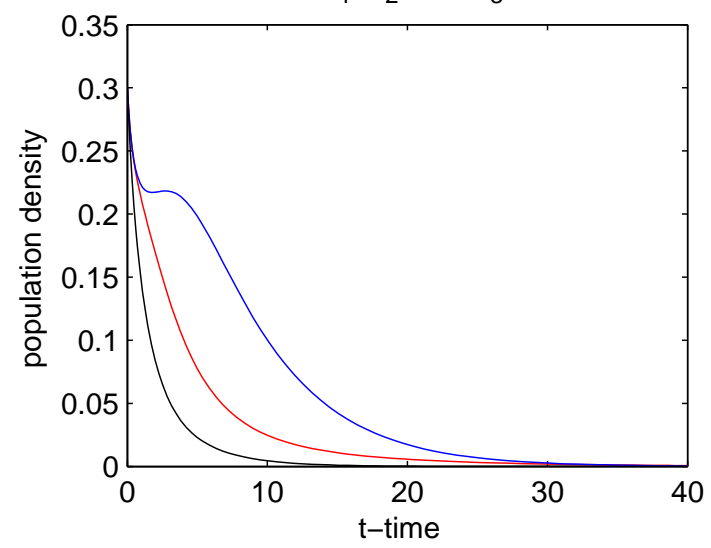

(b) $\mathrm{a}_{1}=\mathrm{a}_{2}=\mathrm{a}_{3}=50$

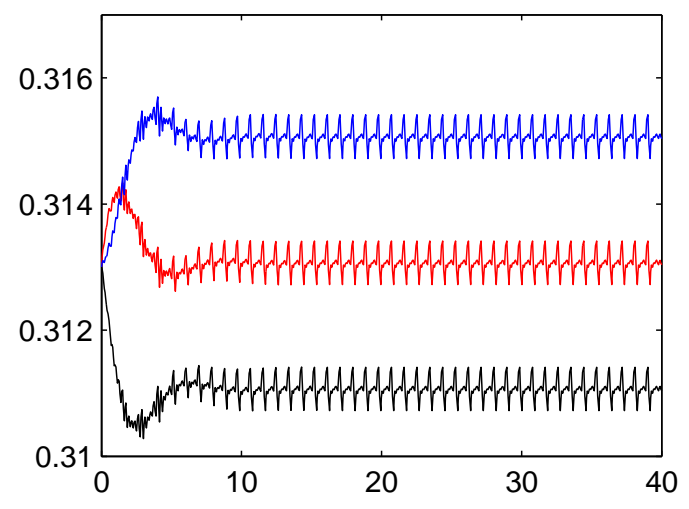

(d) $\mathrm{a}_{1}=\mathrm{a}_{2}=6.5$

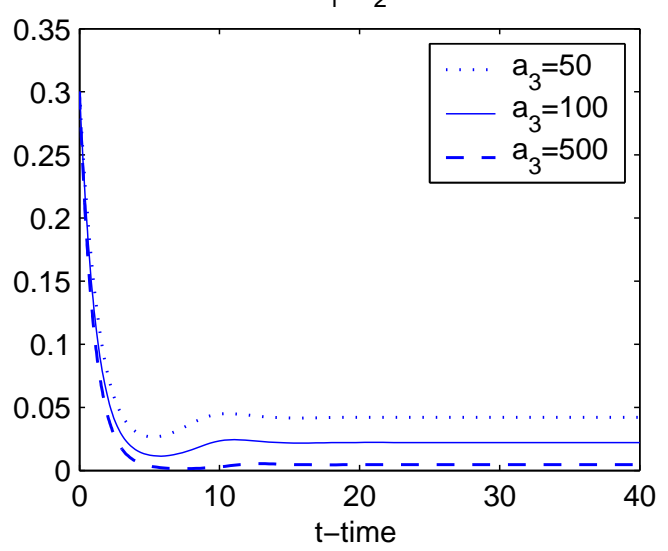

Figure 8: Hopf bifurcation and extreme mutualism in obligate mutualisms. The red, blue and black curves represent solutions of $N_{1}(t), N_{2}(t)$ and $N_{3}(t)$, respectively. Fix $r_{1}=0.9, r_{2}=0.8, r_{3}=0.7, m_{i}=1$ and initial values $N_{i}(0)=0.3, i=1,2,3$. (a) When $a_{i}(=6.5)$ are intermediate, the species coexist at an equilibrium. (b) When $a_{i}(=50)$ are strong, the species coexist in periodic oscillations. (c) When the mutualistic effects $a_{1}=a_{2}(=6.5)$ are large but $a_{3}(=1)$ is extremely small, all species go extinct. (d) Fix $a_{1}=a_{2}=6.5$ and let $a_{3}$ vary. When $a_{3}$ increases from 50,100 to 500 , the density of species $2\left(N_{2}^{+}\right)$decreases monotonically and tends to 0 . 